\title{
A SYSTEM OF AXIOMS FOR GEOMETRY*
}

\author{
BY \\ OSWALD VEBLEN
}

Contents.

Chapter I. The axioms and their independence.

Introductory statement of the axioms . . . . . . . . . $\$ 1$

Categorical and disjunctive systems . . . . . . . . . $\$ 2$

Independence proofs and historical remarks on axioms IX-XII. §3-6

Finite systems proving the independence of I-VIII . . . . $\$ 7-14$

Chapter II. General properties of space.

Definition and properties of line, plane, space . . . . . . \$1-3

Generalizations of order. Regions. . . . . . . . . . \$4

Continuity . . . . . . . . . . . . . . . . . $\$ 5$

Parallel lines . . . . . . . . . . . . . . . . $\$ 6$

Chapter III. Projective and metric geometry.

Ideal elements defined independently of XI and XII . . . . \$1-2

Fundamental theorems of projective geometry . . . . . \$3-6

Definition of congruence relations . . . . . . . . . . §7-8

The axiom system as categorical. . . . . . . . . . $\$ 99$

\section{CHAPTER I. \\ THE AXIOMS AND THEIR INDEPENDENCE.}

$\S 1$. Statement of the axioms.

Euclidian geometry is a system of propositions codifying in a definite way $\dagger$ our spatial judgments. A system of axioms for this geometry is a finite number of these propositions satisfying the following two conditions:

1. Every proposition of euclidian geometry can be deduced from the axioms.

2. No axiom can be deduced from the other axioms.

* Presented to the Society April 11, 1903. Received for publication March 3, 1904.

$\dagger$ By no means the only way. Cf. Bolyai, Lobatchewsky, Veronese. 
Thus an axiom differs from any other proposition of the science, as thus codified, in that it is unproved. *

The propositions brought forward as axioms in this paper are stated in terms of a class of elements called "points" and a relation among points called "order"; they thus follow the trend of development inaugurated by PASCH, $\dagger$

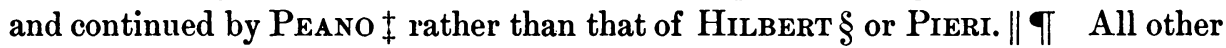
geometrical concepts, such as line, plane, space, motion, are defined in terms of point and order. In particular, the congruence relations are made the subject of definitions** rather than of axioms. This is accomplished by the aid of projective geometry according to the method first given analytically by CAYLEY and KLEIN. $\dagger \dagger$ The terms "point" and "order" accordingly differ from the other terms of geometry in that they are undefined.

The axioms are twelve in number; they presuppose only the validity of the operations of logic and of counting (ordinal number).

Axıом I. There exist at least two distinct points.

Axıom II. $\S \S$ If points $A, B, C$ are in the order $A B C$, they are in the order $C B A$.

Axıom III. If points $A, B, C$ are in the order $A B C$, they are not in the order $B C A$.

\footnotetext{
* Many writers replace the word "axiom" by "unproved proposition" or "primitive proposition."

† M. PASCH, Vorlesungen über neuere Geometrie, Leipzig, Teubner, 1882.

$\ddagger$ G. Peano, I principii di geometria, Turin, 1889; Sui fondamenti della geometria, Rivista di Matematica, vol. 4 (1894), pp. 51-59.

$\S$ D. Hilbert, Grundlagen der Geometrie, Leipzig, 1899. The references are to the English translation by E. J. Townsend, Open Court Publishing Co., Chicago, 1902.

I! M. Pingi, Della geometria elementare come sistema ipotetico deduttivo. Monografia del punto e del moto, Memorie della Reale Accademia delle Scienze di Torino (2), vol. 49 (1899),
} pp. 173-222. For other references to PIERI, see footnote to $\&$ 2, chap. III.

T Particular theorems or axioms derived from these and other writers I have acknowledged in footnotes or in \$§ 3-14 of this chapter. I wish to express deep gratitude to Professor E. H. MOoRE, who has advised me constantly and valuably in the preparation of this paper, and also to Messrs. N. J. Lennes and R. L. Moore, who have critically read parts of the manuscript.

** A definition is an agreement to substitute a simple term or symbol for more complex terms or symbols. On the distinction between definitions and axioms see A. PADoA, Biblothèque du Congrès International de Philosophie, III, "Logique et Histoire des Sciences" (1901), p. 309 ; Peano, ibid., p. 279 ; E. H. Moore, Bulletin of the American Mathematical Society (2), vol. 9 (1903), p. 402. O. Veblen, Monist, vol. 13, no. 2 (January, 1903), pp. 303-9.

†† A. CAYley, Sixth Memoir on Quantics, Collected Works, vol. 2, p. 56 (see also CaYley's note, Ibid., p. 605).

F. KLEIN, Mathematisohe Annalen, vol. 4 (1870), p. 573.

J. Тномак, Geometrie der Lage, Halle, 1873.

E. B. WiLson, Projective and Metric Geometry, A n nals of Mathematics, vol.5, p. 145(1901). $\sharp$ In particular, the distinctness of $A$ and $B$ in the order $A B C$ is a theorem.

\& $\mathrm{Mr}$. R. L. Moone suggests that "If $A$ is a point, $B$ is a point, $C$ is a point" would be a more rigorous terminology for the hypotheses of II, III, IV, inasmuch as we do not wish to imply that any two of the points are distinct. 
Axıom IV. If points $A, B, C$ are in the order $A B C$, then $A$ is distinct from $C$.

Axıом V. If $A$ and $B$ are any two distinct points, there exists a point $C$ such that $A, B, C$ are in the order $A B C$.

Def. 1. The line $A B(A \neq B)$ consists of $A$ and $B$ and all points $X$ in one of the possible orders $A B X, A X B, X A B$. The points $X$ in the order $A X B$ constitute the segment $A B . A$ and $B$ are the end-points of the segment.

Axıом VI. If points $C$ and $D(C \neq D)$ lie on the line $A B$, then $A$ lies on the line $C D$.

Axiom VII. If there exist three distinct points, there exist three points $A$, $B, C$ not in any of the orders $A B C, B C A$, or $C A B$.

Def. 2. Three distinct points not lying on the same line are the vertices of a triangle $A B C$, whose sides are the segments $A B, B C, C A$, and whose boundary consists of its vertices and the points of its sides.

Axiom VIII. If three distinct points $A, B$, and $C$ do not lie on the same line, and $D$ and $E$ are two points in the orders $B C D$ and $C E A$, then a point $F$ exists in the order $A F B$ and such that $D$, $E, F$ lie on the same line.

DEF. 5. A point $O$ is in the interior of a triangle if it lies on a segment, the end-points of which are points of different sides of the triangle. The set of such points $O$ is the

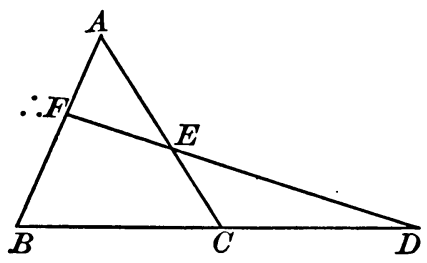

Fig. interior of the triangle.

Def. 6. If $A, B, C$ form a triangle, the plane $A B C$ consists of all points collinear with any two points of the sides of the triangle.

Axıом IX. If there exist three points not lying in the same line, there exists a plane $A B C$ such that there is a point $D$ not lying in the plane $A B C$.

Def. 7. If $A, B, C$, and $D$ are four points not lying in the same plane, they form a tetrahedron $A B C D$ whose faces are the interiors of the triangles $A B C, B C D, C D A, D A B$ (if the triangles exist)* whose vertices are the four points, $A, B, C$, and $I$, and whose edges are the segments $A B$, $B C, C D, D A, A C, B D$. The points of faces, edges, and vertices constitute the surface of the tetrahedron.

DEF. 8. If $A, B, C, D$ are the vertices of a tetrahedron, the space $A B C D$ consists of all points collinear with any two points of the faces of the tetrahedron.

\footnotetext{
* Without this phrase, the definition might be thought to involve some hypothesis about the non-collinea ity of the vertices of the tetrahedron. In $K_{\text {III }}^{\prime}(z 12)$ there is a tetrahedron of only three faces.
} 
Aхгом X. If there exist four points neither lying in the same line nor lying in the same plane, there exists a space $A B C D$ such that there is no point $E$ not collinear with two points of the space, $A B C D$.

Ахгом XI. If there exists an infinitude of points, there exists a certain pair of points $A C$ such that if $[\sigma]^{*}$ is any infinite set of segments of the line $A C$, having the property that each point which is $A, C$ or a point of the segment $A C$ is a point of a segment $\sigma$, then there is a finite subset $\sigma_{1}, \sigma_{2}, \cdots, \sigma_{n}$ with the same property.

Axiom XII. If $a$ is any line of any plane $\alpha$ there is some point $C$ of $\alpha$ through which there is not more than one line of the plane $\alpha$ which does not intersect $a$.

\section{§ 2. Categorical system. Independence of axioms in general.}

Inasmuch as the terms point and order are undefined one has a right, in thinking of the propositions, to apply the terms in connection with any class of objects of which the axioms are valid propositions. It is part of our purpose however to show that there is essentially only one class of which the twelve axioms are valid. In more exact language, any two classes $K$ and $K^{\prime}$ of objects that satisfy the twelve axioms are capable of a one-to-one correspondence such that if any three elements $A, B, C$ of $K$ are in the order $A B C$, the corresponding elements of $K^{\prime}$ are also in the order $A B C$. Consequently any proposition which can be made in terms of points and order either is in contradiction with our axioms or is equally true of all classes that verify our axioms. The validity of any possible statement in these terms is therefore completely determined by the axioms; and so any further axiom would have to be considered redundant. $\dagger$ Thus, if our axioms are valid geometrical propositions, they are sufficient for the complete determination of euclidian geometry.

A system of axioms such as we have described is called categorical, whereas one to which it is possible to add independent axioms (and which therefore leaves more than one possibility open) is called disjunctive. $\neq$ The categorical property of a system of propositions is referred to by HilberT in his "Axiom der Vollständigkeit," which is translated by Townsend into "Axiom of Completeness." E. V. Huntington, $\S$ in his article on the postulates of the real number system, expresses this conception by saying that his postulates are sufficient for the complete definition of essentially a single assemblage. It would probably be better to reserve the word definition for the substitution of one

* [e] denotes a set or class of elements, any one of which is denoted by $e$ alone or with an index or subscript.

$\dagger$ Even were it not dedncible from the axioms by a finite number of syllogisms.

$\ddagger$ These terms were suggested by Profrssor John Dewey.

Z E. V. Huntington, A Complete Set of Postulates for the Theory of Absolute Continuous Magnitude, Transactions of the American Mathematical Society, vol. 3, p. 364 (1902). 
symbol for another, and to say that a system of axioms is categorical if it is sufficient for the complete determination of a class of objects or elements.

An example of a disjunctive set of axioms is furnished as soon as we leave out any one of the axioms in the set above. For instance, all the first eleven axioms are satisfied if the class of points is taken to consist of all the points interior to a sphere and order as referring to the usual relation of order among these points. Axiom XII is evidently not a valid proposition of this class. The first eleven axioms (excluding XII) therefore can refer to at least two essentially different classes and so form a disjunctive system.

The example just given serves also to show that axiom XII satisfies the second of the conditions named in $\S 1$ for a proposition to be an axiom. It cannot be logically deduced from axioms I-XI; in technical language, it is independent.

In the following sections, besides some remarks on the history and formal peculiarities of the axioms, the independence of each of the twelve axioms is to be proved in the manner just indicated. That is, for axiom $N(N=\mathrm{I}, \mathrm{II}, \ldots, \mathrm{XII})$ a class of objects $K_{N}$ will be exhibited which satisfies each of the axioms except $N$ and for which $N$ is not satisfied. Therefore if axiom $N$ were omitted the system of axioms would be disjunctive and so the validity of $N$ is not determined by the other axioms.

\section{§ 3. Parallels. Axiom XII.}

The parallel axiom was the first whose independence was seriously studied. Its history* is so well known that I need only mention the names, BoLYAI, Lobatchewsky, Riemann, Beltrami, Cayley, Klein. The form in which it is here stated is due to C. Burali-Forti. $\dagger$

The class of points interior to a sphere has been given in $\S 2$ as $K_{\mathrm{XII}}$.

The questions whether axiom XII would still be sufficient if stated for only one plane instead of for any plane, or for only one line of any plane instead of for any line of any plane can both be answered in the negative. The class $K_{\mathrm{XII}}^{\prime}$, consisting of all the points on a certain side of a certain plane $\pi$, is such that in at least one plane, namely, any plane parallel to $\pi$, there is only a single parallel to any line through any point. In the same system, in any plane, there is at least one line, namely, the line parallel to $\pi$, to which there is only one parallel through any point.

\section{§4. Continuity. Axiom XI.}

The proposition here adopted as the continuity axiom is referred to by

* For bibliography, see G. B. Halstued, A merican Journal of Mathematics, vol. 7, . p. 264 (1878); also American Mathematical Monthly, vol. 8, pp. 216-230 (1901).

†C. Burali-Forti, Les postulats pour la géométrie d'Euclide et de Lobatchewsky, p. 264, Verhandlungen des Ersten Internationalen Mathematiker Congresses, Leipzig, 1898. 
Schoenflies * as the Heine-Borel theorem. So far as I know, it was first stated formally (as a theorem of analysis rather than of geometry) by BoREL $\dagger$ in 1895 but is involved in the proof of the theorem of uniform continuity given by HeINe $\ddagger$ in 1871 . The idea of its equivalence with the Dedekind cut axiom was the result of a conversation with Mr. N. J. LenNes.

The independence of XI is well known. XI and its consequence, the so-called Archimedean axiom, $\S$ have been discussed by Veronese, $\|$ Stolz, $\uparrow$ LeviCivita, ** Hilbert, $\dagger$ and Dehn.

That theorem 38, which shows the existence of at least one parallel to a given line, cannot be proved without recourse to XI is shown by $K_{\mathrm{XI}}$ of which the points are the rational points of an ordinary coördinate geometry, viz., points whose coördinates are all rational numbers, and also the points at infinity collinear with at least two rational points. To define order, consider the plane $\sigma$ through the points $(\pi, 0,0),\left(0, \pi^{2}, 0\right),\left(0,0, \pi^{3}\right)$ where $\pi$ is the ludolphian constant. This plane contains no point of $K_{\mathrm{XI}}$. Pcints $A, B, C$, of $K_{\mathrm{XI}}$ are in the order $A B C$ if, in the ordinary geometry, $A, B . C$ are collinear and $A, C$ are separated by $B$ and the point in which the line $A B$ meets the plane $\sigma$. The axioms I-X are satisfied and every two complanar lines meet.

$K_{\mathrm{XI}}$ is determined categorically by a system of twelve axioms, the first ten of which are identical with I-X, and the eleventh of which is

$\mathrm{XI}^{\prime}$. On a certain line there are three points that are harmonically related to every point of the line.

and the twelfth of which is

XII'. Every two complanar lines meet in a point.

On the basis of these axioms the same proof of the fundamental theorem of pro-

* A. Schoenflies, Bericht übe- die Mengenlehre, Jahresbericht der Deutschen Mathematiker-Vereinigung, vol. 8 (1898), p. 51.

†É. Borel, Annales de l'École Normale Supérieure (3), vol. 12 (1895), p. 51 . It is stated by BOREL for a numerable set of segments.

$\ddagger$ E. Heine, Die Elemente der Functionenlehre, Crelle, vol. 74 (1872), p. 188.

\& Cf. O. Stolz, Zur Geometrie der Alten, insbesondere über ein Axiom des Archimedes, Mathematische Annalen, vol. 22 (1883).

\|G. Veronese, Fondamenti di geometria, a piu dimensioni e a piu specie di unita rettilinee, Padua, 1891. German translation by Schepp, Leipzig, 1894.

ๆ0.STolz, Ueber das Axiom des Archimedes, Ma th ematisch e A n nal e n, vol. 39 (1891), p. 107.

** T. Levi-Civita, M emorie della Reale Accademiadei Lincei Roma, vol. 7 (1898), pp. 91-96, 113-131.

†† D. HilberT, 1. c., § 12 and also, Ueber den Satz von der Gleichheit der Basiswinkel im gleichschenkligen Dreieck, Proceedings of the Lond on Mathematical Society, vol. 35 (1902), p. 50.

施. Den N, Die Legendre'schen Sätze über die Winkelsumme im Dreieck, Mathematische Annalen, vol. 53 (1900), p. 404.

\& 8 A test of completeness for the axiomatic analysis of a mathematical science would be the question whether for every theorem one can specify a subset of the axioms, sufficient and individually indispensable for its proof. In the present system the answer to this question is affirmative in the cases that $I$ have investigated. 
jective geometry can be made as in $\S 6$, chapter III because theorem 67 is an evident consequence of $\mathrm{XI}^{\prime}$.

\section{§ 5. Tridimensionality. Axiom X.}

As $K_{\mathrm{X}}$ may be taken the usual four-dimensional geometry. The axiom equivalent to $\mathrm{X}$ in the systems of $\mathrm{PASCH}$ and HilberT is :

"If two planes $\alpha, \beta$ have a point $A$ in common, then they have at least a second point $B$ in common." *

This is essentially theorem 25, $\S 3$, chapter II. Peano's axiom XVI in the Principii is nearly the same as ours; namely, " if $A$ and $B$ are such that the segment $A B$ meets a plane $\pi$, then for any point $X$, either the segment $A X$ or the segment $B X$ meets $\pi$."

\section{§6. Relation of plane and space geometry. Axiom IX.}

The class of points $K_{\mathrm{IX}}$ is the set of points in an ordinary plane subject to the usual order relations. The non-desarguesian "geometries" of HILBERT $\dagger$ and Moulton $\ddagger$ may be enumerated respectively as $K_{\mathrm{IX}}^{H}$ and $K_{\mathrm{IX}}^{M}$. They show the essential dependence of the Desargues theorem (62, chap. III) and harmonic properties ( $\$ 5$, chap. III) of the plane upon axiom IX.

This axiom, not stated explicitly by $\mathrm{PASCH}_{\mathrm{AS}}$ is number $\mathrm{XV}$ (page 23) in Peano's Principii, and is part of I7 in Hilbert's system. For the suggestion to state this axiom for only a single plane as well as VII for only a single triad of points I am indebted to Professor Moone.

\section{§7. Significance and construction of finite independence proofs.}

The independence proofs of the first eight axioms can all be exhibited by means of finite classes of elements or points. That such independence proofs are not possible for IX-XII is a consequence of theorem 12, based on I-VIII, that every line contains an infinitude of points. The finiteness of $K_{\mathrm{I}}, \ldots, K_{\mathrm{vIII}}$ shows conversely that every one of the first eight axioms is essential to the proof of theorem 12. It also brings into relief an important difference between our system of axioms and the systems based on RiEmans's concept of space as fundamentally a number-manifold. By the method of RiemanN, the axioms serve to distinguish space from all possible number-manifolds, by the other method they distinguish it from all systems of objects of any kind.

* The form quoted is HilberT's II 6.

† D. Hilbert, Foundations of Geometry, p. 74, § 23.

$\ddagger$ F. R. Moulton, A simple non-desarguesian plane geometry, Transactions of the American Mathematical Society, vol. 3 (1902), p. 192.

Trans. Am. Math. Soc. 23. 
The description of the following tactical systems is preliminary to the exposition of $K_{\mathrm{I}}, \ldots, K_{\mathrm{VIII}}{ }^{*}$

By $S\{3,2,5\}$ is denoted the system of ordered triads or permutations :

$$
\begin{array}{l|l}
123,234,345,451,512 & 135,352,524,241,413 \\
321,432,543,154,215 & 531,253,425,142,314
\end{array}
$$

Each ordered pair of elements determines a unique element that precedes it, a unique element that follows it and a unique middle element. All the triads can be obtained from 123, 321, 531, 135 by the cyclic substitution (12345).

By $S[3,2,7]$ is denoted the triadic system of seven vertically printed triads:

$$
\begin{array}{lllllll}
0 & 1 & 2 & 3 & 4 & 5 & 6 \\
1 & 2 & 3 & 4 & 5 & 6 & 0 \\
3 & 4 & 5 & 6 & 0 & 1 & 2
\end{array}
$$

It has the property $1^{\text {st }}$ that every pair of elements lies in a unique triad and $2^{\text {nd }}$ every pair of triads has a unique common element. $S[3,2,7]$ is invariant under the well-known group $G_{168}^{7}$ of order 168 with generators,

$$
\begin{aligned}
& S_{1}=(0123456), \\
& S_{2}=(0)(124)(365), \\
& S_{3}=(0)(1)(3)(26)(45), \\
& S_{4}=(0)(1)(3)(25)(46) .
\end{aligned}
$$

All the triads are obtained from any one by the cyclic substitution $S_{1}$. Notation apart, this is the only triadic system in seven elements.

Analogous to a triadic system is the pentadic system $S[5,2,21]$ of twenty-one vertically printed pentads :

$\begin{array}{rrrrrrrrrrrrrrrrrrrrr}0 & 1 & 2 & 3 & 4 & 5 & 6 & 7 & 8 & 9 & 10 & 11 & 12 & 13 & 14 & 15 & 16 & 17 & 18 & 19 & 20 \\ 1 & 2 & 3 & 4 & 5 & 6 & 7 & 8 & 9 & 10 & 11 & 12 & 13 & 14 & 15 & 16 & 17 & 18 & 19 & 20 & 0 \\ 6 & 7 & 8 & 9 & 10 & 11 & 12 & 13 & 14 & 15 & 16 & 17 & 18 & 19 & 20 & 0 & 1 & 2 & 3 & 4 & 5 \\ 8 & 9 & 10 & 11 & 12 & 13 & 14 & 15 & 16 & 17 & 18 & 19 & 20 & 0 & 1 & 2 & 3 & 4 & 5 & 6 & 7 \\ 18 & 19 & 20 & 0 & 1 & 2 & 3 & 4 & 5 & 6 & 7 & 8 & 9 & 10 & 11 & 12 & 13 & 14 & 15 & 16 & 17\end{array}$

Every pair of elements lies in one and only one pentad and every two pentads have in common one and only one element. $S[5,2,21]$ is invariant under a group $G^{21}$ of order $21 \cdot 2016 \cdot 9 \cdot 2=120960$, with the following generators :

* A rich collection of such systems (not, however, including $S[5,2,21]$ ) is to be found in the nemoir, Tactical Memoranda, I-III, by E. H. Mooke, A merican Journal of Mathematics, vol. 18 (1896), p. 264. 
$T_{1}=(0,1,2,3,4,5,6,7,8,9,10,11,12,13,14,15,16,17,18,19,20)$, $T_{2}=(0)(1,2,4,8,16,11)(3,6,12)(5,10,20,19,17,13)(7,14)(9,18,15)$, $T_{3}=(0)(1)(2,9)(7,19)(6,8,18)(12,11,16,4,15,3)(5,14,17,10,20,13)$, $T_{4}=(0)(1)(3,16)(17,13)(6,18,8)(2,4,15,9,12,11)(7,10,20,19,5,14)$, $T_{5}=(0)(1)(2)(6)(8)(18)(7,19,9)(12,16,15)(3,20,10)(4,17,14)(5,13,11)$, $T_{6}=(0)(1)(2)(3)(8)(9)(16)(12,15)(13,17)(6,18)(7,19)(5,14)(4,11)(10,20)$.

All the pentads are obtained from any one by the cyclic substitution $T_{1}$. The group is doubly transitive and indeed such that any three elements not belonging to the same pentad can be transformed into $0,1,2$.

\section{§ 8. Triangle transversal. Axiom VIII.}

Axiom VIII was originally stated by $\mathrm{P}_{\mathrm{ASCH}}{ }^{*}$ in a form equivalent to this : "A line lying wholly in a plane and intersecting one side of a triangle of the same plane intersects the perimeter of the triangle in one other point." It was retained by Hilbert as his II 5 in practically the same form. Peano $\dagger$ made of it two axioms (XIII and XIV) from either of which VIII can be deduced. The statement of the axiom was made considerably weaker than any of its previous forms by Professor MooRe, $\ddagger$ and the present form is weaker than his.

As the points of $K_{\mathrm{vIII}}$ we take the twenty-one elements of $S[5,2,21]$. The pentad $0,1,6,8,18$ taken in a definite order determines a system of triads of the type $S\{3,2,5\}$. The order of any pentad being defined as that obtained from the above by a cyclic substitution, the twenty-one pentads determine forty-two systems of triads--420 triads in all.

Three points of $K_{8}$ are in the order $A B C$ if $A B C$ is one of the 420 triads just specified.

Axiom I is valid for $K_{\mathrm{VIII}}$ because there are twenty-one points, and axiom II because the order $A B C$ implies a system $S\{3,2,5\}$ which by its definition includes the triad $C B A$. Axiom III is verified because each triad, and indeed each pair, appears in only one pentad and that pentad determines only one order system of the type $S\{3,2,5\}$ in which each triad can appear only once. IV is equivalent to the statement that each triad consists of distinct elements. To verify $\mathrm{V}$ observe that every pair of elements determines a pentad, and in each pentad by the definition of $S\{3,2,5\}$ each pair of elements has a predecessor and a successor.

* M. PASCh, l. c., p. 21.

† G. Peano, l. c., pp. 18, 21.

$\ddagger$ E. H. Mooke, On the projective axioms of geometry, Transactions of the American Mathematical Society, vol. 3 (1902), p. 147. 
A line in $K_{\mathrm{VIII}}$ consists of the points of a pentad. VI is a consequence of the proposition that any pair of elements is contained in only one pentad. VII is verified by the points $0,1,2$ which appear together in no pentad. Since only one point is between any two points, each side of a triangle in $K_{\text {virI }}$ consists of but one point, and thus a plane consists of at most $3+3 \times 3=12$ points. This verifies IX. $\mathrm{X}$ and XII are consequences of the proposition that $0,1,2$ can be transformed into any non-collinear triad and that every two lines have a point in common. XI is satisfied vacuously, i. e., its hypothesis is not fulfilled.

To show that VIII is contradicted, consider the triangle $A=0, B=1, C=3$. Then $D=13$ and $E=9$. The point of intersection $F$ of the lines $D E$ and $A B$ is 18 , and hence $A, B$, and $F$ are in the order $F A B$.

\section{§9. Axiom VII.}

In the class $K_{\mathrm{VII}}$ there are five points, $1,2,3,4,5$. Points $A, B, C$ are in the order $A B C$ if and only if they constitute a triad $A B C$ in the system. $S\{3,2,5\}$. The verification of I-VI for $K_{\mathrm{VII}}$ was in effect carried out in $\S 8$. VII is negated since all the five points are collinear. VIII-XII are satisfied vacuously.

As $K_{\mathrm{VII}}^{\prime}$ one may take the points of a geometrical line with the usual relations of order.

\section{§10. Axiom VI.}

In the class $K_{\mathrm{VI}}$ there are twenty-six points $P_{1}, P_{2}, P_{3}, P_{4}, P_{5}, 0,1,2, \ldots, 20$. Points $A B C$ are in the order $A B C$ if and only if they constitute a triad in one of the sets of triads,

$$
\begin{aligned}
& P_{1}, P_{2}, P_{3}, P_{4}, P_{5} \text { ordered according to } S\{3,2,5\} \text {, } \\
& 0,1, \ldots, 20 \text { ordered according to } S[5,2,21] \text { as in } \S 8 \text {, } \\
& P_{i} h P_{j} \quad(i, j=1, \cdots, 5 ; i \neq j ; h=0, \cdots, 20), \\
& h P_{i} k \quad(h, k=0, \cdots, 20 ; h \neq k ; i=1, \cdots, 5) .
\end{aligned}
$$

VI is contradicted in $K_{\mathrm{VI}}$ because $K_{\mathrm{VI}}$ includes the orders $0 P_{1} 1$ and $1 P_{1} 2$, without containing 012,120 , or 201 . Since all the twenty-six points lie on the line $P_{1} P_{2}$ the hypotheses of VIII-XII are not fulfilled. I-V are easily verified.

\section{§10. Axiom V.}

$K_{\mathrm{v}}$ consists of the points, 1 and 2 , with the agreement that points $A, B, C$ are in the order $A B C$ if and only if $A \neq B, B \neq C, C \neq A$. I is evidently verified and since there exists no triple of points in the order $A B C, \mathrm{~V}$ is denied. All the other axioms are satisfied vacuously. 


\section{§11. Axiom IV.}

$K_{\text {IV }}$ consists of two points, 1 and 2 with the agreement that points $A, B, C$ are in the order $A B C$ if $A=1, B=2, C=1$, or if $A=2, B=1, C=2$. IV is evidently contradicted, and I, II, III, V, VI are evidently satisfied. VIIXII are satisfied vacuously.

\section{§ 12. Axiom III.}

$K_{\text {III }}$ consists of two points, 1 and 2 with the agreement that points $A, B, C$ are in the order $A B C$ if $A \neq B$ and $B=C$ or if $A=B$ and $B \neq C$.

$K_{\text {III }}^{\prime}$ consists of seven points $0,1,2,3,4,5,6$, with the agreement that points $A, B, C$ are in the order $A B C$ if they are distinct and constitute a triad of the triadic system $S[3,2,7](\S 7)$. In verifying $K_{\mathrm{III}}^{\prime}$ note that a line is a triad of points. The plane 123 consists of the collinear points $4,5,0$. The tetrahedron 0124 has as faces the interiors of the triangles $012,024,041$ (not 124) consisting of the triads $364,165,325$. Thus the space 0124 includes all seven points.

$K_{3}^{\prime \prime}$ consists of the points of the ordinary projective geometry (see chapter III) with the agreement that points $A, B, C$ are in the order $A B C$ if they are distinct and incident with the same projective line.

\section{§ 13. Axiom II.}

$K_{2}$ consists of two points, 1 and 2 , with the agreement that points $A, B$, $C$ are in the order $A B C$ if and only if $A \neq B$ and $B=C$.

\section{§ 14. Axiom I.}

$K_{1}$ consists of one point with the agreement that points $A, B, C$ are in the order $A B C$ if and only if $A \neq B \neq C \neq A$. The other axioms are satisfied vacuously.

\section{CHAPTER II.}

\section{General properties of space.}

\section{§1. Properties of the line.}

Aхгом I. There exist at least two distinct points.

Ахгом II. If points $A, B, C$ are in the order $A B C$, they are in the order $C B A$.

Axıom III. If points $A, B, C$ are in the order $A B C$, they are not in the order $B C A$.

Tн. 1 (II, III).* If points $A, B, C$ are in the order $A B C$, they are not in the order $C A B$.

* The roman numbers in parentheses denote the axioms upon which the proof of the theorem depends. Theorems are occasionally referred to by arabio numbers in parentheses. 
Proof. Since the points $A, B, C$ are in the order $A B C$, that is, for brevity, $A B C$, then by II $C B A$, and hence by III the order $B A C$ cannot exist, and therefore by II $C A B$ cannot.

TH. 2 (II, III). The order $A B C$ implies that $A$ is distinct from $B$ and $B$ from $C$.

Proof. If $A$ were the same as $B$, the orders $A B C$ and $B A C$ would be the same, contrary to theorem 1 . Therefore $A$ is distinct from $B$. On account of II, the same proof shows that $B$ is distinct from $C$.

Aхгом IV. If points $A, B, C$ are in the order $A B C$, then $A$ is distinct from $C$.

Corollary (II, III, IV). The order $A B C$ implies that $A, B$, and $C$ are distinct points.

Aхгом V. If $A$ and $B$ are any two distinct points, there exists a point $C$ such that $A, B$, and $C$ are in the order $A B C$.

If $A$ and $B$ stand for the same point we write $A=B$; if for distinct points $A \neq B$.

Def. 1. The line $A B(A \neq B)$ consists of $A$ and $B$ and all points $X$ in any one of the orders $A B X, A X B, X A B$. The points $X$ in the order $A X B$ constitute the linear segment $A B$, or segment $A B$. They are said to lie between $A$ and $B$ to be points of the segment $A B$ or interior points of the segment $A B ; A$ and $B$ are end-points of the segment. The points $X$ in the order $A B X$ constitute the prolongation of the segment $A B$ beyond $B$, and those in the order $X A B$ the prolongation beyond $A$. The points of a line are said to lie on the line.

In terms of def. 1 the results of the preceding axioms and theorems are:

TH. 3 (I, II, II, IV, V). Every pair of distinct points $A$ and $B$ defines one and only one line $A B$, and one and only one segment $A B$. The line or the segment $A B$ is the same as the line or the segment $B A . A$ and $B$ are not points of the segment $A B$. No two of the three sets of points that (with $A$ and $B$ ) constitute the line $A B$ (namely, the segment $A B$ and its two prolongations), have a point in common. There exists at least one point on each prolongation of the segment $A B$.

It has not been shown as yet that every segment $A B$ contains a point nor that the pair of points $A B$ may not lie on some other line than the line $A B$.

Axıom VI. If points $C$ and $D(C \neq D)$ lie on the line $A B$, then $A$ lies on the line $C D$.

TH. 4 (I-VI). Two distinct points lie on one and only one line.

Proof. By theorem 3, it is necessary only to show that if $C$ and $D$ are any two distinct points of a line $A B$ the line $C D$ is the same as the line $A B$. Suppose first that $D$ is the same as $B$; we have to show that the line $A B$ is the same as the line $B C$. Let $X$ be any point of the line $B C(A \neq X \neq B)$. 
As $A$ is known to be a point of $B C$, it follows from VI that $B$ is a point of the line $A X$. Hence we have one of the orders $A B X, A X B$ or $X A B$, and therefore $X$ is a point of $A B$. Similarly any point of $A B$ is shown to be a point of $B C$. Hence the lines $A B$ and $B C$ are the same. Similar proofs hold if $D=A$ or $C=A$ or $C=B$.

If $D$ and $C$ are both distinct from $A$ and $B$, we argue that the line $A B$ is the same as $B C$, which is the same as $C D$.

Corollary. Two distinct lines can have in common at most one point. Such a common point $F$ is called a point of intersection and the lines are said to intersect in $F^{\prime}$.

Aхгом VII. If there exist three distinct points there exist three points $A, B$, $C$, not in any of the orders $A B C, B C A$, or $C A B$.

Th. 5 (I-VII). If $D E$ is any line, there exists a point $F$ not lying in this line.

Proof. If the line $D E$ contains every point, it would contain the three points $A, B, C$ mentioned in VII. The line $A B$, by theorem 4 , would be the same as $D E$, and hence $A B$ would contain the point $C$, contrary to VIII.

Def. 2. Points lying in the same line are said to be collinear. Three distinct non-collinear points, $A, B$, and $C$, are the vertices of a triangle $A B C$, whose sides are the segments $A B, B C, C A$, and whose boundary consists of its vertices and the points on its sides.

Axıом VIII (Triangle transversal axiom). If three distinct points $A, B, C$ do not lie on the same line, and $D$ and $E$ are two points in the order $B C D$ and $C E A$, then a point $F$ exists in the order $A F B$, and such that $D, E, F$ lie on the same line.

TH. 6 (I-VIII). Between every two distinct points there is a third point.

Proof. Let $A$ and $B$ be the given points (fig. 2). By theorem 5 there is a point $E$ not lying on the line $A B$. By $\mathrm{V}$ points $C$ and $D$ exist, satisfying the order-relations $A E C$ and $B C D$. Hence, by VIII, $F$ exists in the order $A F B$.

Tн. 7 (I-VI, VIII). The points $D, E, F$, of axiom VIII, are distinct, and lie in the order $D E F$.

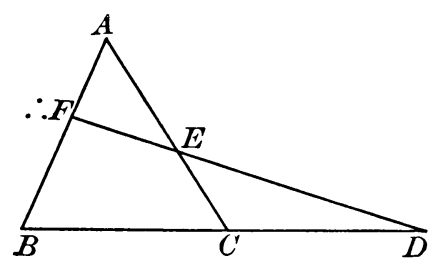

Fig. 2.

Proof. If $D, E, F$ were not distinct, we should by axiom VI have $A, B, C$ collinear. Hence there must be one of the three orders $D E F, E F D, F D E$, and it is necessary to show $E F D$ and $F D E$ impossible.

1st. Suppose the points were in the order $E F D$ (fig. 3). Consider the triangle $D E C$ ( $D, E, C$ being non-collinear by $\mathrm{VI})$, and the orders $A E C$, $D F E$. By VIII, a point $X$ exists on the line $A F$ and in the order $D X C$. But as this point is common to the lines $A F$ and $D C$, which have $B$ in common, by the corollary of theorem $4, X=B$. Ilence we have the order $D B C$ contrary to hypothesis. Hence $D F E$ is impossible. 
2d. Suppose $E D F$ (fig. 4). Consider the triangle $E F A$ ( $E, F, A$ being non-collinear by VI) and the orders $A E C$ and $E D F$. By the same argument as above, we find that $B$ is on the line $C D$ and in the order $A B F$, which contradicts our hypothesis. Hence $E D F$ is impossible.

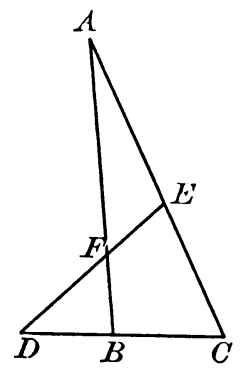

- FIG. 3.

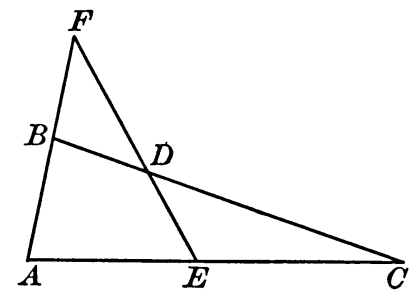

FIg. 4.

Th. 8 (I-VI, VIII). If points $A, B, C$ form a triangle, there is no line that has a point in common with each of the sides $A B, B C, C A$.

Proof. If the theorem is not verified, we have three collinear points $A^{\prime}, B^{\prime}$, $C^{\prime}$, in the three orders $A B^{\prime} C, B C^{\prime} A$, and $C A^{\prime} B$. It is evidently sufficient to show the order $A^{\prime} B^{\prime} C^{\prime}$ impossible.

If $A^{\prime} B^{\prime} C^{\prime}$, then $A^{\prime}, C^{\prime}$, and $B$ would form a triangle; for otherwise $A, B, C$ would be collinear. In connection with the triangle $A^{\prime} C^{\prime} B$, consider the order relations $B A^{\prime} C, A^{\prime} B^{\prime} C^{\prime}$. By theorem 7, a point $X$ exists in the order $B X C^{\prime}$, and $C B^{\prime} X$. Hence $X$ is the intersection of the lines $A B$ and $B^{\prime} C$. Hence $X=A$. Hence $B A C^{\prime}$ contrary to hypothesis.

DEF. 3. If points $A, B, C, D$ are in the orders $A B C, A B D, A C D, B C D$, they are said to be in the order $A B C \cdot D$.

TH. 9 (I-VIII).* To any four distinct points of a line, the notation $A, B$, $C, D$, may always be assigned so that they are in the order $A B C D$.

Proof. The proof of this theorem depends on the following six lemmas:

1. If $A B C$ and $B C D$, then $A B D$.

By $\mathrm{V}$ and theorem 5 , there exist points $P$ and $O$ not in the line $A B$ but in the order $B P O$ (Fig. 5). By VIII and theorem 7 , since we have the triangle $O B C$ and the orders $A B C$ and $B P O$ there exists a point $Q$ in the orders $O Q C$ and $A P Q$. Like-

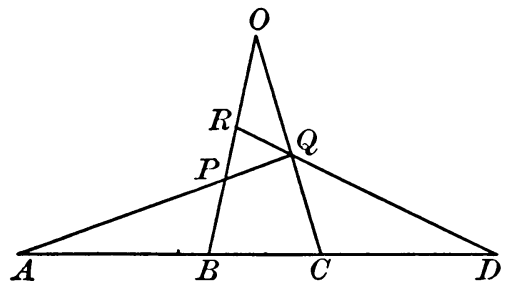

FIG. 5.

* This proposition was given as "Axiom II 4" in HinberT's Festschrift (p. 7). Its redundancy as an axiom of HilberT's system I, II was proved by E. H. MoorE, On the projective axnoms of geometry, Transactions of the American Mathematical Society, Jauuary, 1902, vol. 3, p. 142-168, 501. A second proof has heen given by Mr. R. L. MoorE, cf. p. 98, American Mathematical Monthly, April, 1902. 
wise, since we have the triangle $O B C$ and the orders $B C D$ and $O Q C$, there exists a point $R$ in the orders $O R B$ and $D Q R$. Finally, since we have the triangle $A Q D$, and the orders $R Q D$ and $A P Q$, there exists a point $X$ in the orders $A X D$ and $R P X$. But the intersection of the lines $A D$ and $R P$ is $B$. Hence by the corollary to theorem $4, X=B$. Hence $A B D$.

2. If $A B C$ and $A B D$ then either $B C D$ or $B D C$.

In consequence of VI it is only necessary to show the order $C B D$ impossible. By (5) and $\mathrm{V}$ there exist points $O$ and $P$ not collinear with $B$ and $C$ and in the order $O C P$ (fig. 6). Therefore unless our theorem is valid, we have a triangle $O C D$ and the orders $O C P$ and $C B D$. Hence by VIII and (7), there exists a point $Q$ in the orders $O Q D$ and $P B Q$. Now we have the tri-

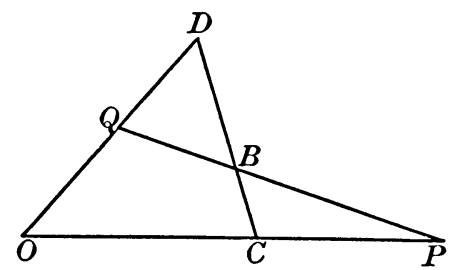

Fig. 6.

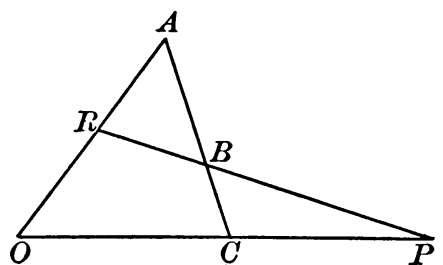

FIG. 7.

angle $A C O$ (fig. 7) and the orders $O C P$ and $A B C$. Hence a point $R$ exists in the orders $P B R$ and $A R O$. From the orders $P B R$ and $P B Q$ it follows by VI that $R, B$, and $Q$ are collinear. But $R, B$, and $Q$ are points of the sides $O A, A D$, and $D O$, of the triangle $O A D$, and this is in contradiction with theorem 8. Hence $C B D$ is impossible.

3. It follows from 1 and 2 that if $A B C$ and $A B D$, then either $A C D$ or $A D C$.

4. If $A B D$ and $A C D$, then either $A B C$ or $A C B$.

By theorem 4, we must have either $A B C$ or $A C B$ or $B A C$. In the last case, from $B A C$ and $A C D$ we could by 1 argue $B A D$, contrary to hypothesis

5. If $A B C$ and $A C D$, then $B C D$.

By theorem 4, if not $B C D$ then $B D C$ or $C B D$. In the first case, $D C A$ leads by lemma 1 to $B C A$-a contradiction; and in the second case, $C B A$ leads by lemma 3 to $C A D$ or $C D A$, either of which contradicts $A C D$.

6. If $A B C$ and $A C D$, then $A B D$.

By lemma 5 we have $B C D$, and hence $A B C$ leads by lemma 1 to $A B D$.

We are now ready to complete the proof of theorem 9. Assign the notation $A, B, C$ to three of the points so that they are in the order $A B C$. The fourth point $D$ will either lie in the order

a) $D A B$, in which case it follows from 1 that we have $D A B C$.

b) $A D B$, in which case it follows from 5, 6 that we have $A D B C$.

c) $A B D$, in which case it follows from 2,1 that we have $A B C D$ or $A B D C$. In any case the notation can be assigned so that the order is $A B C D$. 
Def. 4. The order $A_{1} A_{2} \cdots A_{l} \cdots A_{n}$ means that the points $A_{i}, A_{j}, A_{k}$ are in the order $A_{i} A_{j} A_{k}$ if $i<j<k(i, j, k=1,2, \ldots, n)$.

Tн. 10 (I-VIII). To any finite number $n$, of distinct points of a line, the notation can be so assigned that they are in the order $A_{1} A_{2} \cdots A_{l} \cdots A_{n}$. Proof. By mathematical induction.

TH. 11 (I-VIII). Any finite set of $n$ distinct points separates a line into $n-1$ segments and two prolongations of segments, no two of which have a point in common.

Proof. It is a consequence of theorem 3 that one point separates a line into two mutually exclusive parts, and two points into three mutually exclusive parts. One point separates a segment into two mutually exclusive segments (9); and a prolongation into a segment and a prolongation. Hence if $m$ points separate a line into $m-1$ segments and two prolongations, adding one point adds one segment, and therefore $m+1$ points divide a line into $m$ segments and two prolongations. Hence by induction from the case $m=2$ the theorem is proved.

Th. 12 (I-VIII). Between any two points $A$ and $B(A \neq B)$ and also on either prolongation of the segment $A B$, there exist an infinitude of points.

Proof.* By theorems 6 and 9 it can be proved that if $n$ is any integer there are more than $n$ points $X$ in the order $A X B$ or $A B X$.

\section{$\S 2$. Properties of the plane.}

TH. 13 (I-VIII). $\dagger$ If $A, B$, and $C$ form a triangle, and $D$ and $F$ exist in the orders $B C D$ and $A F B$, then $E$ exists in the orders $A E C$ and $D E F$. Proof (fig. 8). By V and (9) there exists a point $O$ in the order $O B F A$.

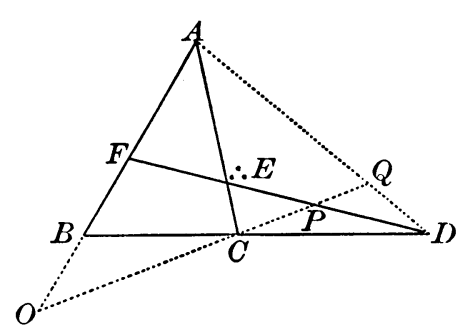

Fig. 8. Hence by VIII and (7), from the triangle $D B F$ and the orders $O B F$ and $B C D$, we obtain a point $P$ in the orders $O C P$ and $F P D$, and from the triangle $D A F$ and the orders $O F A$, $F P D$ a point $Q$ in the orders $O P Q$ and $A Q D$. By (9), we then have $O C P Q$. Hence in the triangle $C A Q$ we have $A Q D$ and $C P Q$. Hence $E$ exists in the order $A E C$, and on the line $P D$, whence by (7) $E$ is in the order $D E F$.

TH. 14 (I-VIII). A line that intersects one side of a triangle and a prolongation of another side intersects the third side.

Proof. This simply combines in one statement VIII and theorems 7 and 13.

* That all of the axioms I-VIII are necessary for the proof of this theorem is demonstrated in \& 8 7-14, chap. I.

$\dagger$ This proposition has been regarded as an axiom in previous discussions; cf. $\S 8$, chap. I. 
DeF. 5. A point $O$ is in the interior of a triangle if it lies on a segment, the end-points of which are points of different sides of the triangle. The set of such points $O$ is the interior of the triangle.

TH. 15 (I-VIII). If $O$ is a point collinear with two points $M$ and $N$ of the perimeter of a triangle $A B C$, a line joining $O$ to any point $P$ of a side of the triangle meets the perimeter in a point $Q(Q \neq P)$.

Proof. This is easily seen if $O$ lies on one of the lines $A B, B C$, or $C A$, or if $M$ or $N$ is a vertex; in the latter case, if $M O N$, by joining $O A, O B$, and $O C$.

Thus we may suppose that $M$ and $N$ are interior points of $A B$ and $A C$ respectively. In case of the order $M N O$ (figs. 9 and 10), considering the

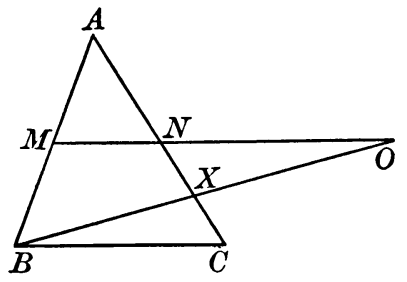

Fig. 9.

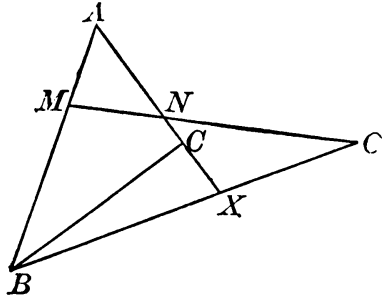

FIG. 10.

triangles $O M B$, we see that $X$ exists in the orders $B X O$ and $A N X$, and in view of the order $A N C$, we have either $A X C$ or $A C X$. In either case the theorem reduces to theorem 14 , in connection with the triangles $A B X$ and $B X C$.

In case of the order $M O N$ (fig. 11), we have by consideration of the triangles $M N B$ and $B N C$ a point $X$ in the order $A O X$ and $B X C$. Likewise, a point $Y$ in the orders $B O Y$ and $C Y A$, and a point $Z$ in the orders $C O Z$ and $A Z B$. If $P$ is a point of $B C$, the theorem follows (14) by consideration of the triangle $A B X$ or $B X C$. A similar argument holds if $P$ is a point of $A B$ or $A C$.

Def. 6. If $A, B, C$ form a triangle, the plane $A B C$ consists of all points collinear with any two points of the sides of the triangle.

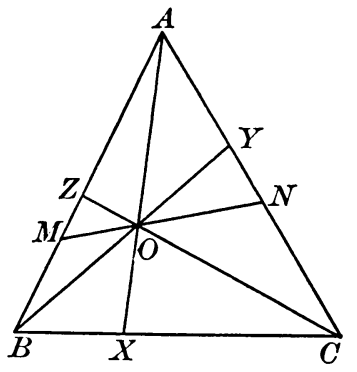

Fig. 11. TH. 16 (I-VIII). Any three non-collinear points lying in a plane determine the plane.

Proof. Let the given plane be $A B C$. If $O$ is any point of a side $A B$ of the triangle $A B C$, the points of the plane $A B C$ consist of the points of the lines joining $O$ to the perimeter of $A B C$. This is a corollary of theorem 15 . We prove : 
1st, that if $A^{\prime}$ is any point of the line $A B$, such that $A^{\prime} \neq B$, the plane $A B C$ is the same as the plane $A^{\prime} B C$. In the case of the order $A^{\prime} A B$ or $A A^{\prime} B$ (fig. 12), we choose $O$ so that it lies between $A$ or $A^{\prime}$ and $B$, and the proposition is an evident consequence of theorem 14. In case of the order $A B A^{\prime}$, we have just shown that the plane $A B C$ is the same as $A A^{\prime} C$, which is the same as $B A^{\prime} C$ or $A^{\prime} B C$.

2nd, that if $C^{\prime}$ is any point of the plane $A B C$ not collinear with $A$ and $B$, the plane $A B C$ is the same as $A B C^{\prime}$. Take $P$ between $A$ and $B$ and join

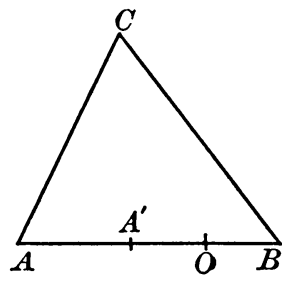

FIG. 12.

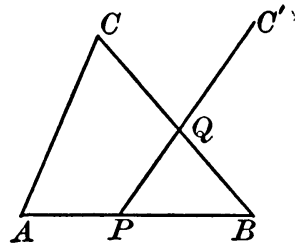

Fia. 13.

$P C^{\prime}$ (fig. 12), meeting the perimeter of $A B C$ in a point $Q$, say $Q=C$ or $Q$ on the side $B C$. By the second part of the proof, $A B C$ is the same as $P B C$, and hence as $P Q B$, and hence as $P C^{\prime} B$, and hence as $A C^{\prime} B$.

3rd, the second step shows that if $A^{\prime} B^{\prime} C^{\prime}$ are any three non-collinear points of the plane, the notation $A^{\prime} B^{\prime} C^{\prime}$ being assigned so that $A^{\prime}$ is not in $B C$ and $B^{\prime}$ is not in $A^{\prime} C, A B C$ is the same as $A^{\prime} B C$, hence the same as $A^{\prime} B^{\prime} C$, and hence as $A^{\prime} B^{\prime} C^{\prime}$.

Corollary. A line lying wholly in a plane $\pi$ and having a point in common with the interior or one side of a triangle which also lies in the plane $\pi$ meets the perimeter of the triangle in two points.

TH. 17 (I-VIII). A line having two points in common with a plane lies wholly in the plane.

Proof. Let the two points be taken as $A$ and $B$ in defining the plane, $A B C$. The plane contains the line $A B$.

Corollary. If two planes have two points in common they have a line in common.

\section{§ 3. Properties' of space.}

Aхıом IX. If there exist three points not lying in the same line, there exists a plane $A B C$, such that there is a point $D$ not lying in the plane $A B C$.

Tн. 18 (I-IX). If $A B C$ is any plane, there exists a point $D$ not in this plane.

Proof. If the plane contained all points it would contain the points $A, B$, 
$C$ mentioned in IX, and would therefore by theorem 16 be the same as the plane $A B C$ of IX, and that plane would contain all points which is contrary to IX. DeF. 7. If $A, B, C, D$ are four points, not lying in the same plane, they form a tetrahedron $A B C D$ whose faces are the interiors of the triangles $A B C, B C D, C D A, D A B$; whose vertices are the four points $A, B, C$, $D$; and whose edges are the six segments $A B, B C, C D, D A, A C$, $B D$. A point $X$ is in the interior of the tetrahedron if it lies between two points $D$ and $E$ of different faces. The points of the faces, edges, and vertices constitute the surface of the tetrahedron.

TH. 19 (I-IX). If any vertex $A$ of a tetrahedron $A B C D$ is joined to an interior point $O$, the line $A O$ meets the opposite face $B C D$ in a point $P$ lying in the order $A O P$.

Proof. Since $O$ is an interior point, there exist two points $X$ and $Y$ of the surface in the order $X O Y$ (fig. 14). These two points are not both in the same

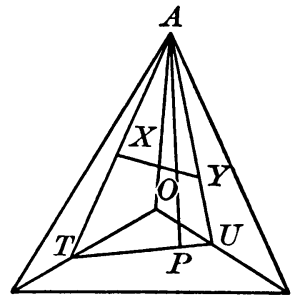

Fig. $\cdot 14$.

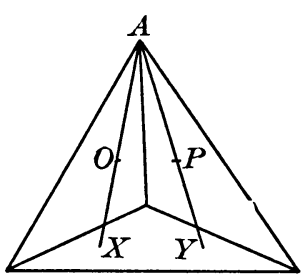

Fig. 15.

face of the tetrahedron, and therefore one of them, $X$ is in a face that has $A$ for a vertex. Join $A X$, and if $Y$ is in a face that has $A$ for a vertex, join $A Y$ also. $A X$ and $A Y$ will then meet the perimeter of $B C D$ in two points, $T$ and $U$. In the triangles $X Y T$ and $T Y U$ we have the condition of theorem 14. Hence the required point $P$ exists.

If $Y$ is in the face $B C D$, we have a point $T$ of the perimeter of $B C D$ in the order $A X T$. Then by theorem $15 U$ exists on the perimeter of $B C D$ in the order $T Y U$. But in the triangle $T X Y$ we again have the hypothesis of theorem 14 and hence a point $P$ in the order $T P Y$. In view of the order $T Y U$, $P$ is in the order $T P U$.

TH. 20 (I-IX). If any interior point $O$ of a tetrahedron $A B C D$ is joined to another point $P$ of the surface or interior of the tetrahedron, the line $O P$ meets the surface in two points $Q$ and $R$ (where if $P$ is of the perimeter, $P=Q$ ) lying in the order $Q O R$.

Proof. By theorems 13 and $19, X$ and $Y$ exist on the interior or perimeter of $B C D$ in the order $A O X$ and $A P Y$ (fig. 15). Then, by theorem 13, T and $U$ exist on the perimeter of $B C D$ in the orders $T X U$, and, if $Y \neq U, T Y U$. Hence by $\S 2 O P$ meets the perimeter of the triangle $A T U$, and hence the surface of the tetrahedron, in the required points $Q$ and $R$. 
Tн. 21 (I-IX). If $O$ is a point in the order $M N O$ with two points $M$ and $N$ of different faces of a tetrahedron $A B C D$, the line joining $O$ to an interior point $P$ of a face of the tetrahedron meets the surface in a point $Q, Q \neq P$.

Proof [I-IX]. 1st (fig.16). Suppose that $P$ is on the same face with $M$ or $N$. If with $M$, let $X$ be a point of this face in the order $M P X$. Then, (14), $O P$ meets the segment $N X$ in a point $Y . Y$ is an interior point of the tetrahedron, and hence the existence of $Q$ follows from theorem 20. A similar argument holds if $P$ is on the same face with $N$.

2d (fig. 17). Suppose that $P$ is not on the same face with $M$ or $N$. Let $A$ be the vertex not in the same face with $P$. Since $A$ is a vertex of every

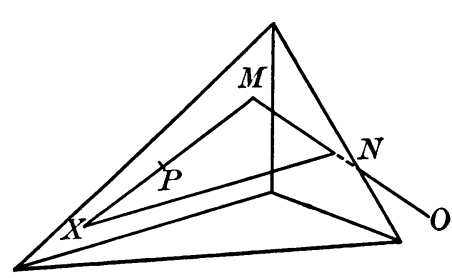

FIG. 16.

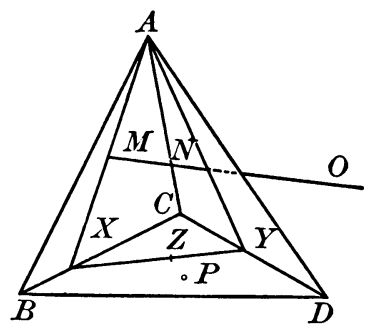

Fia. 17.

face but $B C D$, on joining $A M$ and $A N$ we obtain $X$ and $Y$ as points of the perimeter of $B C D$ in the orders $A M X$ and $A N Y$. Hence by theorem 15 the line joining $O$ to any point $Z$ in the order $X Z Y$ meets the perimeter of the triangle $A X Y$ in a point $W$. That $P O$ meets the perimeter now follows from the first part of this proof if $O$ is not in the plane $B C D$ and from $\S 2$ if $O$ is in the plane $B C D$.

DEF. 8. A space $A B C D$, if $A, B, C$, and $D$ form a tetrahedron, consists of all points collinear with two points of the faces of the tetrahedron.

TH. 22 (I-IX). If two points of a line lie in a space $A B C D$, so does every point of the line.

Proof. If the two points are both complanar with one of the faces of the

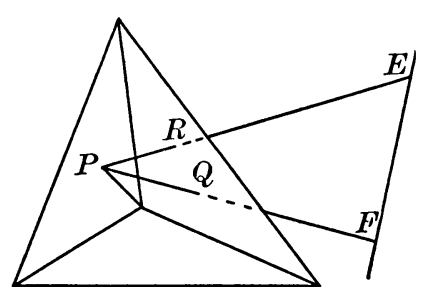

FIG. 18. tetrahedron $A B C D$, apply the results of $\S 2$. If not, let the two points in question be $E$ and $F$ (fig. 18). By theorems 20 and 21 , if $P$ is a point of one face, not collinear with $E$ and $F, Q$ and $R$ exist on the surface of $A B C D$, such that $Q$ is on the line $P F$ and $R$ on the line $P E$, and $P, Q$, $R$ are not collinear. $F$ and $E$ lie in the plane $P Q R$, and therefore any point of the line $X$ can be joined to two points of the perimeter of the triangle $P Q R$. But every point of $P Q R$ is on the surface or in the interior of $A B C D$, and therefore by theorems 20 and 21 every point of the line $F E$ lies in the space $A B C D$. 
Tн. 23 (I-IX). Any four non-complanar points of a space define that space.

Proof. Let the given space be $A B C D$, and the four non-complanar points be $A^{\prime}, B^{\prime}, C^{\prime}, D^{\prime}$. By theorem 22 every point of the lines $A^{\prime} B^{\prime}$ and $A^{\prime} C^{\prime}$ belongs to $A B C D$. Hence every point of the plane $A^{\prime} B^{\prime} C^{\prime}$, and hence every point of the space $A^{\prime} B^{\prime} C^{\prime} D^{\prime}$. Similarly, every point of $A B C D$ belongs to the space $A^{\prime} B^{\prime} C^{\prime} D^{\prime}$.

Corollary. If three points of a plane lie in a space, so does every point of the plane.

Ахıом X. If there exist four points neither lying in the same line nor lying in the same plane, there exists a space $A B C D$ such that there is no point $E$ not collinear with two points of the space $A B C D$.

TH. 24 (I-X). There is but one space.

Proof. By theorem 22 and 23 and axiom $\mathrm{X}$.

Tн. 25 (I-X). Two planes that have a point in common have a line in common.

Proof. It is sufficient under the corollary to theorem 17 to show that two planes $\alpha$ and $\beta$ have two points in common. If $O$ is the given point, let the plane $\alpha$ be defined by points $A_{1}, A_{2}, A_{3}$, such that $O$ is an interior point of the triangle $A_{1} A_{2} A_{3}$ (this is possible by 16). If $B$ is a point of the second plane not lying in $\alpha$, let space be defined by the tetrahedron $B A_{1} A_{2} A_{3}$ (fig. 19). If $B^{\prime}$ is a third point of $\beta$ the line $B^{\prime} O$, which lies wholly in $\beta$, meets the surface of the tetrahedron (by 24,20,21), in another point,

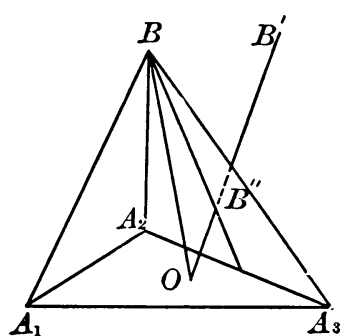

FIG. 19. $B^{\prime \prime}$. If this point $B^{\prime \prime}$ is in $\alpha$, the theorem is proved; if not, join $B B^{\prime \prime}$. The line $B B^{\prime \prime}$ lies in $\beta$ and meets one of the lines $A_{1} A_{2}, A_{2} A_{3}, A_{3} A_{1}$ in a point common to $\alpha$ and $\beta$.

\section{§4. Generalizations of order. Regions.}

Def. 9. The points lying on a system of segments $A_{1} A_{2}, A_{2} A_{3}, \cdots, A_{n-1} A_{n}$ together with the points (called vertices) $A_{1}, A_{2}, \ldots, A_{n}$ constitute a broken line. If $A_{n}=A_{1}$, the broken line, $A_{1}, \ldots, A_{n}$ is the boundary of a polygon of which $A_{i}(i=1, \ldots, n-1)$ are the vertices and the segments $A_{i} A_{i+1}$ are the sides. A multiple point of a polygon or broken line is a point common to a side and a vertex or to two sides or to two vertices. The boundary of a polygon without multiple points is called simple.*

DEF. 10. A region is a set of points any two of which are points of a broken line composed entirely of points of the set. Two regions, $R^{\prime}$ and $R^{\prime \prime}$, sub.

* A polygon is any figure (i. e. class) of sides and vertices, the points of which constitute the " boundary of a polygon." Similar distinctions are made between " angle" and "boundary of an angle," "trihedral angle" and " boundary of a trihedral angle," etc. 
sets of the same region, $R$, are separated with respect to $R$ by a set of points $[S]$ if every broken line of $R$ joining a point of $R^{\prime}$ to a point of $R^{\prime \prime}$ contains at least one point of the set $[S] \cdot[S]$ is said to decompose $R$ into a finite number $n$ of regions if there are $n$ and only $n$ regions $R^{\prime}, R^{\prime \prime}, \ldots, R^{(n)}$ that include every point of $R$ except $[S]$ and if every pair of regions $R^{(s)}$ and $R^{(k)}$ are separated by $[S]$. Lines, planes, and space are obvious examples of regions as is also the interior of a triangle; the latter is referred to as a triangular region.

TH. 26 (I-VIII). Any line decomposes a plane in which it lies into two regions.

Proof. Let $A$ be a point of the line $a$, and $A^{+}, A^{-}$two points of the plane, $\alpha$, not of $a$, and in the order $A^{+} A A^{-}$. It is to be proved: (1) that a region including ${ }_{A^{+}}^{A^{+}}$and including no point of $a$ does not include ${ }_{A^{+}}^{A^{-}} ;(2)$ that any region of the plane that includes all points that can be joined to a given point by broken lines not meeting $a$ includes either $A^{+}$or $A^{-}$.

The statement (1) is a consequence of the proposition that any broken line of the plane joining $A^{+}$to $A^{-}$has a point in common with $a$. If this were not so a certain broken line $A^{+} B_{1} B_{2} \cdots B_{n} A^{-}$would not intersect $a$. By the corol. lary to theorem 16 , since $A^{+} B_{1}, B_{1}$, and $B_{1} B_{2}$ have no point in common with $a$, neither would $A^{+} B_{2}$. By $n-1$ such steps it appears that $A^{+} B_{i}(i=2 \cdots n)$ would have no point in common with $a$ and hence $A^{+} A^{-}$would not meet $a$.

The proof of statement (2) depends on the observation that any point $P$ of $\alpha$ but not of $a$ is such that one and only one of the segments $P A^{+}$and $P A^{-}$ meets $a$. Otherwise the triangle $P A^{+} A^{-}$would meet the line $a$ only in the point $A$ or else in points of all three sides.

Corollary 1 (I-VIII). A line that meets the boundary of a simple polygon but does not meet any of its vertices meets the boundary of the polygon in an even number of points.

Corollary 2 (I-IX). Any plane $\alpha$ decomposes a space in which it lies into two regions.

DEF. 11. If $A$ and $B$ are two distinct points, the half-line $A B$ consists of the segment $A B, B$, and the prolongation of $A B$ beyond $B$. If $A, B, C$ are three non-collinear points, the half-plane $(A B, C)$ is the one of the two regions, into which the line $A B$ decomposes the plane $A B C$, that contains $C$. The two half-planes determined by a line in any plane are called the two sides of the line. If $A, B, C$ are non-collinear, the boundary of the angle $B A C$ consists of $A$ and the points of the half-lines $A B$ and $A C$. The interior of the angle consists of all points in any region of the plane that does not include the boundary and does include a point $X$ satisfying the order relation $P X Q$ where $P$ is a point of the half-line $A B$ and $Q$ of the half-line $A C$.

TH. 27 (I-VIII). Two non-intersecting complanar lines (if such exist) decom- 
pose their plane into three regions, two intersecting lines decompose it into four regions, and the boundary of an angle decomposes it into two regions.

Proof. Call the two regions into which a line decomposes a plane + and - ; and then apply the method of theorem 26 to the + and - regions separately. (The new regions will be conveniently designated by,,,.+++--+-- )

Corollary 1. In the case of an angle one of the two regions specified by theorem 27 is the interior of the angle. The boundary of an angle meets the boundary of any polygon in its plane and having no vertex or multiple point on the boundary of the angle in an even number of points.

Corollary 2. The boundary of a triangle decomposes the plane in which it lies into two regions, one of which is the interior (def. 5). The other region, which contains lines not intersecting the boundary of the triangle, is called the exterior. Tн. 28 (I-VIII). The boundary of a simple polygon lying entirely in a plane $\alpha$ decomposes $\alpha$ into two regions.

Proof. The proof depends upon two lemmas.

Lemma 1. If a side of a polygon $q$ intersects a side of a polygon $p_{n}$ in a single point $O$ not a multiple point of $p_{n}$ or $q$, then $p_{n}$ and $q$, whether simple or not, have at least one other point in common. If $n=3$ ( $q$ having any number of sides, $m$ ) the theorem reduces to corollary 2 , theorem 27 . We assume without loss of generality that no three vertices $P_{i-1}, P_{i}, P_{i+1}$, are collinear and prove the lemma for every $n$ by reducing to the case $n=3$. Let $p_{n}$ have $n$ vertices with the notation such that the side $P_{1} P_{2}$ meets $q$ in the side $Q_{1}^{\prime} Q_{2}^{\prime}$ where the segment $Q_{2}^{\prime} O$ contains no interior point of the triangle $P_{1} P_{2} P_{3}$. By the case $n=3, q$ meets the boundary of the triangle $P_{1} P_{2} P_{3}$ in at least one point other than $O$. If this point is on the broken line $P_{1} P_{2} P_{3}$ the lemma is verified. If not, $q$ has at least one point on $P_{1} P_{3}$, and at least one of the segmeuts $Q_{1}^{\prime} Q_{2}^{\prime}, Q_{2}^{\prime} Q_{3}^{\prime}$ has no point or end-point on $P_{1} P_{3}$. Let this segment be one segment of a broken line $Q_{k} Q_{k+1} \cdots Q_{j-1} Q_{j}$ of segments of $q$ not meeting $P_{1} P_{3}$ but such that $Q_{k-1} Q_{k}$ and $Q_{j} Q_{j+1}$ do each have a point or endpoint in common with $P_{1} P_{3} . \quad\left(1 \leqq k<j \leqq m\right.$; if $k=1, Q_{k-1}=Q_{m}$; if $\left.j=m, Q_{j+1}=Q_{1}\right)$. If $O_{j}$ is the point common to $P_{1} P_{3}$ and $Q_{j} Q_{j+1}$ or $Q_{j+1}$, and $O_{k}$ the point common to $P_{1} P_{3}$ and $Q_{k-1} Q_{k}$ or $Q_{k-1}$, the broken line $O_{k} Q_{k} Q_{k+1} \cdots Q_{j-1} Q_{j} O_{j}$, has a point inside and also a point outside the triangle $P_{1} P_{2} P_{3}$ and cuts the broken line $P_{1} P_{2} P_{3}$ only once. Hence it has a point inside and a point outside any triangle of which $P_{1} P_{3}$ is a side. On this account if $P_{1} P_{3} P_{4}$ are not collinear, and obviously, if $P_{1} P_{3} P_{4}$ are collinear, $q$ must meet either $P_{3} P_{4}$ or $P_{4}$ or $P_{4} P_{1}$. If $q$ does not meet $P_{3} P_{4}$ or $P_{4}$, we proceed with $P_{1} P_{4} P_{5}$ as we did with $P_{1} P_{3} P_{4}$. Continuing this process, we either verify the lemma or come by $n-2$ steps to the triangle $P_{1} P_{n-1} P_{n}$ and'find that $q$ must intersect the broken line $P_{n-1} P_{n} P_{1}$, which also verifies the lemma. 
Lemma 2. A point $P$ of the boundary of a polygon $p_{n}$ lying in a plane $\alpha$ is said to be accessible from a point $O$ of $\alpha$ if there exists a broken line joining $O$ to $P$ and not meeting the boundary of the polygon in any point other than $P$. Every point of the boundary of a simple polygon $p_{n}$ is accessible from every point $O$ of $\alpha$, not on the boundary of $p_{n}$. That there is at least one point of $p_{n}$ accessible from $O$ is seen by considering any line through $O$ meeting the boundary of $p_{n}$ but not meeting a vertex of $p_{n}$. This line meets $p_{n}$ in a finite number of points and hence in a point $P^{\prime}$ of a side $P_{j} P_{j+1}$ such that the segment $O P^{\prime}$ contains no point of $p_{n}$.

If $P^{\prime}$ is any point of a side $P_{i} P_{i+1}$ of $P_{n}$ accessible from $O$ by means of a broken line $O Q_{1} Q_{2} \cdots Q_{n} P^{\prime}$, it is evident that $P_{i}$ is accessible from $O$ by means of a similar broken line. For the lines joining $P_{i}$ to each of the other vertices of $p_{n}$ meet $Q_{n} P^{\prime}$ in at most a finite number of points. There is, therefore, a point $Q^{\prime}$ such that the segment $Q^{\prime} P^{\prime}$ contains none of these points and thus the segment joining $P_{i}$ to a point of $Q^{\prime} P^{\prime}$ does not meet $p_{n}$ in other points than $P_{i}$.

Considerations similar to those just adduced show that if $P^{\prime \prime}$ is any point of a side $P_{i} P_{i+1}$ such that $P_{i}$ is accessible from $O, P^{\prime \prime}$ is also accessible. Now since $P^{\prime}$ of $P_{j} P_{j+1}$ is accessible from $O$, so is every point of $P_{j} P_{j+1}$ and also $P_{j+1}$, and hence every point of $P_{j+1} P_{j+2}$, and so on for the whole polygon.

If $p_{n}$ is any polygon of $\alpha$, let $A$ and $B$ be two points such that the segment $A B$ intersects a side $P_{1} P_{2}$ but does not meet any other point of the boundary of $p_{n}$. By lemma 1 , every broken line joining $A$ to $B$ meets $p_{n}$. Hence the boundary of any plane polygon whatever decomposes its plane into at least two regions.

If the boundary of a simple polygon $p_{n}$ should decompose $\alpha$ into three regions, let $P$ be a point of the side $P_{1} P_{2}$. $P$ being accessible from each region, there are three segments belonging to different regions whose mutual end-point is $P$. Two of these segments $P C$ and $P D$ must lie in the same one of the two halfplanes defined by $P_{1} P_{2} . C^{\prime}$ and $D^{\prime}$ being points of $P C$ and $P D$ such that no line joining two vertices of $p_{n}$ meets $P C^{\prime}$ or $P D^{\prime}$, it is evident that $C^{\prime} D^{\prime}$ does not meet $p_{n}$ and hence $C^{\prime}$ and $D^{\prime}$ belong to the same region, contradicting the hypothesis of three regions. The boundary of a simple polygon therefore decomposes a plane in which it lies into two and only two regions.

The proofs of the following theorems of this section are similar to the preceding, and therefore are omitted.

DEF. 12. If $A, B, C, D$ are four non-complanar points, the boundary of the dihedral angle of the half-planes $(A B, C)$ and $(A B, D)$ consists of the line $A B$ and the half-planes $(A B, C)$ and $(A B, D)$. If $A B, A C$, $A D$ are three non-complanar half-lines, the boundary of the trihedral angle $(A, B C D)$ consists of the point $A$ (the vertex), the half lines $A B$, 
$A C, A D$ (the edges) and the interiors of the angles $B A C, C A D$. $D A B$ (the faces).

Tн. 29 (I-IX). Two non-intersecting planes (if such exist) decompose a space in which they lie into three regions, two intersecting planes decompose it into four regions, and a dihedral angle or a trihedral angle decomposes it into two regions.

DEF. 13. The interior of a dihedral angle is that region of the two defined by theorem 29 which includes a point $X$ in the order $P X Q$ where $P$ is a point of one of the half-planes of the boundary and $Q$ a point of the other. The interior of a trihedral angle $(A, B C D)$ is that of the two regions defined by theorem 29 which includes a point $X$ in the order $P X Q$ where $P$ is a point of the edge $A B$ and $Q$ of the side $C A D$. The exterior of a dihedral or trihedral angle is that one of the two regions defined by theorem 29 which is not interior.

TH. 30 (I-IX). A plane through the vertex and an interior point of a trihedral angle meets the boundary in two half-lines of which one may be an edge and the other interior to a side or each may be interior to a side different from the side to which the other is interior.

DeF. 14. Let a finite or infinite set of complanar lines $\ldots a \ldots b \ldots c \ldots d \ldots k \ldots$ pass through a point $O$. Let $M$ and $N$ be two points of any line $a$ in the order $M O N$. Let $C$ be any point of a line $c$. If the lines $\ldots b \ldots$ meet $M C$ in a set of points $\ldots B \ldots$ in the order $M \ldots B \ldots C$ and the lines $\ldots d \ldots k \ldots$ meet $C N$ in a set of points $\ldots D \ldots K \ldots$ in the order $C \ldots D \ldots K \cdots N$, then the set of lines is in the order $a \ldots b \cdots c \ldots k \ldots$ In the special case of the order $a b c d, a$ and $c$ are said to separate $b$ and $d$.

Tr. 31 (I-VIII). The above definition is independent of the triangle $M N P$. The notation can be permuted cyclically or reversed. From $a \cdots b \cdots c \cdots k$ and $a b k l$, follows $a \ldots b \cdots c \ldots k l$. If $a$ and $c$ separate $b$ and $d, a$ and $b$ do not separate $c$ and $d$ nor do $a$ and $d$ separate $b$ and $c$. From $a b c d$ and $a b c e(d \neq e)$ follows either abcde or abced. In case of the order $a b c d$, any segment joining a point of $a$ to a point of $c$ meets one or other of the lines $b$ and $d$. Any segment joining a point of $a$ to a point of $b$ either meets both $c$ and $d$ or neither.

Def. 15. Let a finite or infinite set of planes $\alpha \cdots \beta \cdots \gamma \cdots \lambda$ pass through a line $o$. Intersect $o$ by a plane $\pi$. Then if $a$ is the intersection of $\alpha$ and $\pi, b$ of $\beta$ and $\pi$, etc., and the lines $a \ldots b$, etc., are in the order $a \cdots b \cdots c \ldots l \cdots$, the planes are in the order $\alpha \cdots \beta \cdots \gamma \cdots \lambda \ldots$.

TH. 32 (I-IX). The above definition is independent of the plane $\pi$ and analogous statements to those of theorem 30 hold for the planes $\alpha, \beta$, etc. 


\section{§5. Continuity.}

Axiом XI. If there exists an infinitude of points, there exist a certain pair of points $A C$ such that if $[\sigma]$ is any infinite set of segments of the line $A C$, having the property that each point which is $A, C$, or a point of the segment $A C$ is a point of a segment $\sigma$, then there is a finite sub-set $\sigma_{1}, \sigma_{2}, \cdots, \sigma_{n}$ with the same property.

TH. 33 (I-VIII, XI). If $P Q$ is any segment and $[\sigma]$ any infinite set of segments of the line $P Q$, having the property that each point which is $P, Q$, or a point of the segment $P Q$ is an interior point of a segment $\sigma$, then there is a finite subset $\sigma_{1}, \sigma_{2}, \ldots, \sigma_{n}$ having the same property.

Proof. In case of the order $A P Q C$ consider $A^{\prime} P$ and $Q C^{\prime}$ (where $A^{\prime}$ and $C^{\prime}$ are in the order $\left.A^{\prime} A C C^{\prime}\right)$ as segments of $[\sigma]$ and the theorem is a corollary of XI.

In case $P Q$ is not on the line $A C$ but $P=A$, let $O$ be a point in the order $O C Q$. If $[R]$ is the set of those end points of segments $\sigma$ that lie between $P$ and $Q$, by $\S 3$ each of the lines $O R$ meets $A C$ in a point $B$. Thus every segment $\sigma$ which lies between $P$ and $Q$ corresponds to a segment $\sigma^{\prime}$ between $A$ and $C$. To any segment $\sigma$ whose end points $S_{1} S_{2}$ are in the order $P S_{1} Q S_{2}$ let correspond $D_{1} D_{2}=\sigma^{\prime}$ where $D_{1}$ is the point of intersection of $O S_{1}$ with $A C$ and $D_{2}$ any point in the order $A C D_{2}$. Similarly in the case $Q S_{1} P S_{2}$.

By XI there is a finite subset of $\left[\sigma^{\prime}\right], \sigma_{1}^{\prime}, \sigma_{2}^{\prime}, \ldots, \sigma_{n}^{\prime}$ of which $A, C$, and every point of $A C$ are interior points. Any set of $\sigma$ 's corresponding to $\sigma_{1}^{\prime}, \ldots, \sigma_{n}^{\prime}$ is the required set $\sigma_{1}, \cdots, \sigma_{n}$.

If $P=A$ and $Q$ is any point of the line $A C$ let $Q^{\prime}$ be any point not on the line $A C$. The theorem holds for $P Q^{\prime}$ by the preceding paragraph and hence by a similar argument for $P Q$. If $P \neq Q$ and $P Q$ is any segment not of the line $A C$, the theorem follows by the intermediation of the segment $A P$.

TH. 34 (I-VIII, XI). If a segment $A B$ consists of two sets of points $[P]$ and $[Q]$ such that no point $P$ is between two points $Q$ and no point $Q$ between two points $P$ then there exists one and only one point $O$ in the order $P O Q$ for every pair of points $P$ and $Q(P \neq O, Q \neq O)$.

Proof. Supposing the conclusion of the theorem not valid assign the notation so that there is the order $A P Q B$; then including $A$ there is a segment not including any point $Q$ and including $B$ there is a segment not including any point $P$; including every point $P$ there is a segment including no point $Q$ and including every point $Q$ a segment including no point $P$. Of this set of segments $[\sigma]$ there is (33) a finite sub-set including $A, B$, and every point of $A B$. Assign notation to those end points of segments of this set which include points $P$ so that there is the order $A_{1} A_{2} \cdots A_{m} A P_{1} P_{2} \ldots P_{n}$; and to those end points of segments of this set which include points $Q$ so that there is the order $Q_{l} \ldots Q_{2} Q_{1} B B_{l} \cdots B_{1}$. 
By hypothesis we have

$$
A_{1} A_{2} \cdots A_{m} A P_{1} P_{2} \cdots P_{n} Q_{k} Q_{k-1} \cdots Q_{2} Q_{1} B B_{l} \cdots B_{1} .
$$

One of the set of segments whose $n+m+k+l$ end points are here enumerated must include points between $P_{n}$ and $Q_{k}$ but such a segment includes both points of $P$ and of $Q$, contrary to the hypothesis about [ $\sigma]$.

That there is only one point $O$ is shown by remarking that if there were two points, $O$ and $O^{\prime}$, in the order $P O O^{\prime} Q$ for every $P, Q$ there would (6) be a point $S$ in the order $O S O^{\prime}$ and hence in the orders $P S O^{\prime}$ and $O S Q$. But $S$ must be either a $P$ or a $Q$. In the first case the hypothesis would be contradicted by $O S Q$ and in the second case by $P S O^{\prime}$.

DEF. 16. A point $P$ is a limit point of a set of points $[S]$ of a line $A B$ if every segment of $A B$ which contains $P$ contains some point of $[S]$ distinct from $P$.

The following two theorems can be proved either directly from theorem 33 or in the well-known way from theorem 34 .

Tн. 35 (I-VIII, XI). Every infinite set of points lying on a segment has at least one limit point.

TH. 36 (I-VIII, XI). If $A$ and $C$ are any two points and a set of points $[B]$ is in the order $A B C$ there is a point $P$ in the order $A B P$ for every $B \neq P$, and such that if $Q$ is any point in the order $A B Q$ for every $B$, either $P=Q$ or $A P Q$.

TH. 37 (I-VIII, XI). If all the lines through a point $C$, in a plane $\alpha$, consist of two sets $[s]$ and $[t]$ such that each set contains at least two lines, and no two lines of one set separate any two lines of the other set, then there are two lines $o_{1}$ and $o_{2}$ that separate every $s$ $\left(\neq o_{1}, \neq o_{2}\right)$ from every $t\left(\neq o_{1}, \neq o_{2}\right)$. Proof. Let $s_{1}$ and $s_{2}$ be two lines of $[s]$, and $A$ any point of $s_{1}$, while $B$ and $D$ are points of $s_{2}$ in the order $B C D$. By $\S 4$, supposing that some $t$ intersects $A D$ there is no $t$ that intersects $A B$. Let $E$ be the point of intersection of some line $t_{0}$ with $A D$. The theorem follows at once as a corollary of

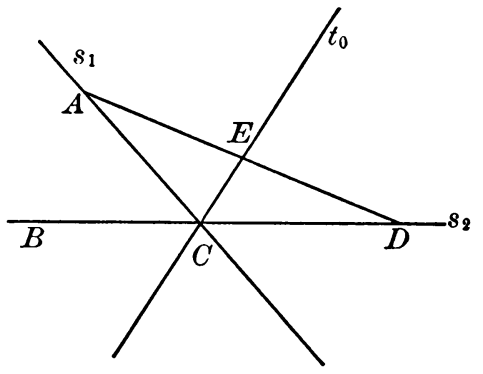

FIG. 20. theorem 34 , by considering the two sets of points of intersection of $[s]$ and [ $t$ ] with the segments $A E$ and $E D$ respectively.

\section{§ 6. Parallel lines.}

TH. 38 (I-VIII, XI). If $a$ is any line of a plane $\alpha$, through any point $C$ of $\alpha$ there is at least one line of $\alpha$ that does not intersect $a$. 
TH. 39 (I-VIII, XI). If there is more than one such line, there are two lines, $c_{1}$ and $c_{2}$, through $C$, not meeting $a$ that separate the lines that do not intersect $a$ from those that do intersect $a$.

Proof of 38.* Let $Q$ and $R$ be any two points of $a$, and let [s] be the set of all lines through $C$ that meet $a$ in points $S$ in the order $Q R S$. Let $[t]$ be the set of all other lines through $C$. No two lines $s$ separate two lines $t$; for in that case one of the lines $t$ would intersect $a$ on the prolongation of $Q R$ beyond $R$. Hence (37) there are two lines $b_{1}$ and $b_{2}$ that separate every line $s$ from every line $t$. Not both of these lines meet $a$. For if this were so, call their points of intersection $B_{1}$ and $B_{2}$ and the points of intersection of an $s$ line, $S$, and of a $t$ line, $T$. If there is a point $T$ in the order $B_{1} T B_{2}$, there are points $S^{\prime}$ and $S^{\prime \prime}$ in the order $S^{\prime} B_{1} B_{2} S^{\prime \prime}$, and hence a $T$ point between two $S$ points, - a contradiction with the hypothesis that all points of the prolongation of $Q R$ are $S$ points. If there is a point $S$ in the order $B_{1} S B_{2}$, there are points $T^{\prime}$ and $T^{\prime \prime}$ in the order $T^{\prime} B_{1} B_{2} T^{\prime \prime}$ and hence in the order $T^{\prime} S T^{\prime \prime}$ and therefore a $T$ point on the prolongation of $Q R$ beyond $R$.

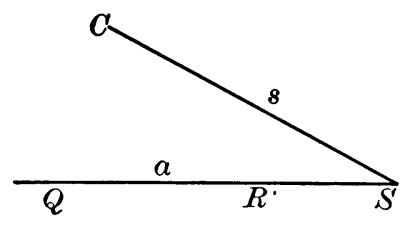

Fig. 21.

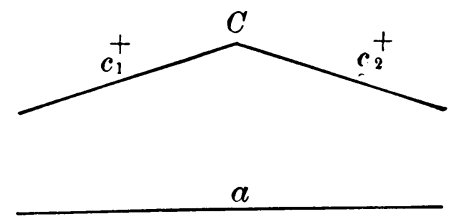

FIG. 22.

Proof of 39. Let all the lines through $C$ that meet $a$ be $[s]$ and all that do not $[t]$. Then the existence of $c_{1}$ and $c_{2}$ follows at once from theorem 37 . $c_{1}$ or $c_{1}$ cannot intersect $a$; for in that case we should have $S_{1} C_{1} S_{2}$ or $S_{1} C_{2} S_{2}$. DeF. 17. In case there are two lines $c$ and $c_{1}$ the line $a$ lies wholly in one of the four regions into which the plane is decomposed by $c_{1}$ and $c_{2}$. Call the two half-lines, $c_{1}^{+}$and $c_{2}^{+}$, that bound this region of the plane the half-lines parallel to $a . \quad a$ and the two parallel half-lines $c_{1}^{+}$and $c_{2}^{+}$separate from the rest of the plane a region of the plane which lies between them and consists of all points that can be joined by a segment not meeting $a$ or $c_{1}^{+}$ or $c_{2}^{+}$to a point $B$ in the order $C B Q$. In case the lines $c_{1}$ and $c_{2}$ coincide in a line $c$, that line is called the parallel to $a$.

Tн. 40 (I-IX). If $a$ has two parallel half-lines, any line through a point between $a$ and its parallel half-lines, $c_{1}^{+}$and $c_{2}^{+}$, meets either $a$ or $c_{1}^{+}$or $c_{2}^{+}$.

Proof. Let $O$ be a point not in the plane $\alpha$. The planes $O c_{1}$ and $O c_{2}$ and $O a$ meet in three lines, $O C, a_{1}$, and $a_{2}$. Let the line through a point between $a$ and $c_{1}^{+}$and $c_{2}^{+}$be $b$. Then, the plane $O b$, passing through the interior of the trihedral angle whose vertex is $O$, meets two of the sides or one side and one edge.

\footnotetext{
* That XI is essential for this theorem is proved by $K_{\mathrm{XI}}, \& 4$, chap. I.
} 
Hence there is at least one line $o^{\prime}$ common to the plane $O b$ and a side of the trihedral angle, and by the lemma below, $o^{\prime}$ meets $a$ or $c_{1}^{+}$or $c_{2}^{+}$.

Lemma. If $o^{\prime}$ is any line through $O$ in the face $O a$ of the trihedral angle, $o^{\prime}$ meets $a$; for the plane $C o^{\prime}$ meets $\alpha$ in a line $a$ that passes through a point in the interior of the angle between $c_{1}^{+}$and $c_{2}^{+}$. If $o^{\prime \prime}$ is any line through $O$ in the face $O c_{1}^{+}$, it meets $c_{1}^{+}$; for joining $o^{\prime \prime}$ to a point $C^{\prime}$ of the half-line of $c_{2}$ other than $c_{2}^{+}$, this plane meets $O a$ in a line $o^{\prime}$ that must intersect $a$ in a point $A$. As the points $A$ and $C$ are on opposite sides of $c_{1}^{+}$, the plane $o^{\prime \prime} C^{\prime}$ meets $c_{1}^{+}$, and therefore $o^{\prime \prime}$ meets $c_{1}^{+}$.

TH. 41 (I-IX). If in a plane $\alpha$ through a point $C$ there is only one line $c$ parallel to $a$, any line of $\alpha$ that meets one of the lines $a$ and $c$ meets the other.

Proof. It follows as in the lemma to theorem $\mathbf{4 0}$ that if $O$ is a point outside $\alpha$, and $o$ the intersection of $O c$ and $O a$, any line of one of these planes that passes through $O$ and is distinct from $o$ meets $a$ if it is in $O a$ and $c$ if it is in $O c$.

If $a^{\prime}$ is a line through a point $A$ of $a$ the plane $O a^{\prime}$ meets $O c$ in a line $o^{\prime}$ distinct from $o$, and $o^{\prime}$ meets $c$ by the first paragraph. Similarly, if $c^{\prime}$ is a line through a point $C^{\prime}$ of $c$, the plane $O c^{\prime}$ meets $O a$ in a line $o^{\prime}$ distinct from $o$, and hence $o^{\prime}$ meets $a$.

TH. 42 (I-IX, XI). If $a$ is a line of a plane $\alpha$ and through one point $C$, not on $a$, there is only one line $c$ that does not intersect $a$, then through any point $C^{\prime}$ of $\alpha$ not on $a$ there is only one parallel to $a$.

Proof. Suppose that through a point $C$ not between $a$ and $c$ there are two parallels $c_{1}$ and $c_{2}$ to $a$. These lines are also parallel to $c$ : for by theorem 41 every line through $\vec{C}$ that meets $c$ also meets $a$, and every line that meets $a$ meets $c$. Then either $c$ lies between $a$ and $c_{1}^{+}$and $c_{2}^{+}$, or $a$ lies between $c_{1}^{+}$and $c_{2}^{+}$and c. Either case is in contradiction with theorem 40. Hence there can be only one parallel through $\bar{C}$. The theorem now follows for any point $\overline{\bar{C}}$ between $a$ and $c$ by considering with $a$ a parallel, on the opposite side of $a$ from $\vec{C}$.

Axıом XII. If $a$ is any line of any plane $\alpha$ there is some point $C$ of $\alpha$ through which there is not more than one line of the plane $\alpha$ which does not intersect $a$.

TH. 43 (I-IX, XI, XII). In any plane $\alpha$ through any point $A$ there is one and only one line parallel to a given line $a$.

Proof. By XII and theorems 38 and 42.

\section{CHAPTER III.}

\section{Projective Geometry.}

\section{§1. Preliminary theorems.}

The development of projective geometry from the foundation established in the preceding chapter $\$ \$ 1-6$, follows methods that are fairly well known. 
On this account and for lack of space the proofs of most of the theorems will be omitted. While it will not often be possible to give a reference which furnishes complete details of a demonstration, the citation will generally cover the methods.

Projective geometry is independent of the parallel axiom and so in this chapter no use will be made of $\S 6$, chapter II before $\S 7$ where the euclidean metrical geometry is defined. In the present $\S 1$ are stated some preliminary theorems from which are derived, in the following $\S 2$, definitions of the projective elements analogous to those originally proposed by KLEIN. * In neither of these sections is any use made of the continuity axiom XI.

Lemma. Let $\pi$ and $\rho$ be two planes intersecting in a line $a$, and let $l$ and $m$ be two lines of $\rho$ lying on the same side of $a$. Then if $l$ is complanar with each of two lines $b$ and $c$ of $\pi$, and $m$ is complanar with $b$, it follows that $m$ is complanar also with $c$.

TH. 44 (I-X). If three lines, $a, b$, and $c$, of a plane $\pi$, are each complanar with a line $l$ not of the plane $\pi$, and $a$ and $b$ are each complanar with another line $m$, then $c$ is also complanar with $m$.

TH. 45 (I-X). If $a$ and $b$ are two lines in a plane $\pi$, and $l$ and $m$ two lines not in $\pi$ but each complanar with $a$ and $b$, then $l$ and $m$ are complanar.

DeF. 18. Two lines $a$ and $b$ lying in the same plane $\pi$, define a system of lines consisting of every line of intersection of a plane through $a$ with a plane through $b$ as well as all the lines in $\pi$ that are complanar with one of the lines of the system that does not lie in $\pi$. Such a system of lines is called a bundle. The system of all lines in a plane $\pi$ complanar with a line $l$ not in $\pi$ is called a pencil.

Tн. 46 (I-X). Every two lines of a bundle are complanar and define the same bundle. Through any point of space passes one line of a given bundle. Any two points $B$ and $C$ not in the same line of a bundle $A$ define with $A$ a plane including all lines of $A$ passing through points of the line $B C$. Two distinet bundles have in common at most one line.

DeF. 19. If $A$ and $B$ are two bundles, through every point $O$ of space there passes one line of each bundle. If these lines do not coincide they define a plane. The system of planes thus defined by two bundles is called a pencil of planes. Every line of $A$ or $B$ not common to $A$ and $B$ lies in one and only one plane of the pencil. A bundle every one of whose lines lies in a plane of the pencil is said to be incident with the pencil.

A special case of a pencil of planes is the set all of planes through a line. TH. 47 (I-X). If $A_{1} B_{1} C_{1}$ and $A_{2} B_{2} C_{2}$ are two triangles of a plane $\pi$, such

* F. Klein, Mathematische Anna!en, vol. 6 (1873), p. 112. Further details are given by M. PASCH, l.c., pages 40 to 72, and by F. SCHUR, Ueber die Einführung der sogenannten idealen Elemente in die projective Geometrie, Mathematische Annalen, vol. 39 (1891), p. 113. A very elegant exposition is due to R. BonoLA, Sulla introduzione degli enti improprii in geometria projettiva, Giornale di Matematiche, vol. 38 (1900), p. 105. 
that the three bundles defined by the three pairs of sides $A_{1} B_{1}$ and $A_{2} B_{2}$, $B_{1} C_{1}$ and $B_{2} C_{2}, C_{1} A_{1}$ and $C_{2} A_{2}$, each has a line in a certain plane $\sigma$, $(\sigma \neq \pi)$ then the joints of corresponding vertices, $A_{1} A_{2}, B_{1} B_{2}, C_{1} C_{2}$, lie in the same pencil.

TH. 48 (I-X.) If a pencil of planes defined by two bundles $L_{1}$ and $L_{2}$ meets a plane $\pi$ in three lines, $a, b$, and $c$, these three lines lie in the same pencil of lines.

Corollary 1. Any bundle having a line in each of two planes of a pencil is incident with the pencil.

Corollary 2. Any two bundles incident with a pencil of planes determine that pencil.

TH. 49 (I-X). If $A B C$ and $A_{1} B_{1} C_{1}$ are two triangles of a plane $\pi$ such that the lines $A A_{1}, B B_{1}, C C_{1}$, are in the same pencil, then the bundles defined by pairs $A B, A_{1} B_{1}, B C, B_{1} C_{1}$, and $A C, A_{1} C_{1}$, lie in the same pencil of planes.

Corollary. If two triangles $A B C$ and $A^{\prime} B^{\prime} C^{\prime}$ are so situated in a plane that the lines joining their corresponding vertices $A A^{\prime}, B B^{\prime}, C C^{\prime}$, meet in a point $P$, and the corresponding sides $A B, A^{\prime} B^{\prime}$, etc., meet in three points, $C_{1}, C_{2}, C_{3}$ then $C_{1}, C_{2}, C_{3}$ are collinear; and conversely.

TH. 50 (I-X). If three bundles, $A, E$, and $C$ lie in one pencil of planes, whereas three bundles $B, C$, and $D$ lie in another pencil of planes, then there is a bundle $F$ incident at once with the pencils $A B$ and $D E$.

\section{§2. The projective elements.}

DEF. 20. A projective point is a bundle of lines.

DEF. 21. A projective line is a pencil of planes. Three points incident with the same line are collinear.

DEF. 22. A projective plane is the set of all projective points collinear with the projective point $O$ and any point of a projective line $o$ not incident with $O$ (including $O$ and the points incident with $o$ ). Any point of the plane is said to be incident with the plane.

DEF. 23. A projective point is called proper if the bundle of lines defining it meet in a point. A projective line or plane is proper if there is incident with it a proper point; otherwise it is improper or ideal.

It will immediately become evident that a proper projective point has the same incidence relations as a point in the sense of chapter II. For the rest of this chapter we shall mean by "point" a projective point, and by "proper point" a proper projective point.

By corollary 2, theorem 48 , any two points incident with a line determine that line. Let a plane $\pi$ be determined by a line $a$ and point $B$ not incident with $a$. That any other point and line incident with $\pi$ determine it, is an immediate 
deduction from theorem 38. This amounts to saying that any three points incident with $\pi$ determine it, and that any line, two of whose points are incident with $\pi$ is also incident with $\pi$. It follows that any two lines incident with $\pi$ are incident with one, and only one, common point.

A line incident with a proper point is by def. 23 and by theorem 25 incident with another proper point, and hence the ordinary line defined by these points is contained in the projective line. Consequently, every plane incident with a with a proper point is incident with three non-collinear proper points, and therefore the ordinary (chapter II) plane determined by these three points is included in the projective plane.

By def. 21, every two proper planes are incident with one, and only one line, proper or improper. A proper plane $\lambda$ and an improper $\mu$ are incident with one and only one improper line. For let $m$ be any line of $\mu$; it is by definition the intersection of two proper planes $\alpha$ and $\beta$ through two proper points $A$ and $B$ of $\lambda$ that are therefore incident with two proper lines $a$ and $b$ of $\lambda . \quad a$ and $b$ define a point at once incident with $m$ and $\lambda$. Hence every line $m$ of $\mu$ is incident with a point $L$ of $\lambda$. But as three non-collinear points determine a plane, these points $L$ must be incident with a line, which proves our proposition.

Finally, two improper planes, $\rho$ and $\sigma$, are incident with one and only one line. For a proper plane $\alpha$ is incident with one line of each of them, so that the point incident with each of these lines is incident with each of the planes $\rho$ and $\sigma$. Another proper plane $\beta$ gives another point, and just as in the preceding paragraph, these two points determine the one and only one line incident with $\rho$ and $\sigma$.

As obvious results of the above argument, we have:*

TH. 51 (I-X). Two points are incident with one and only one line.

Two planes are incident with one and only one line.

TH. 52 (I-X). Three points not incident with the same line are incident with one and only one plane.

Three planes not incident with the same line are incident with one and only one point.

Тн. 53 (I-X). A line and a point not incident with it are incident with one and only one plane.

A line and a plane not incident with it are incident with one and only one point.

TH. 54 (I-X). Two lines incident with the sa. a plane are incident with one and only one point.

* No attempt is here made to ohtain an independent system of basal theorems for projective geometry. The foundations of projective geometry as a science by itself have been studied by M. PIERI, Sui principii che reggono ln geometria della retta, Atti della Reale Accademia della Scienze di Torino, vol. 36 (1901), p. 335 . I principii della y ometria di posizime..., Memorie della Reale Accademia della Scı. 4 e di Torino, vol. 48 (1899), p. 1. 
Two lines incident with the same point are incident with one and only one plane.

Tн. 55 (I-X). If two points are incident with a plane, any line incident with each of them is incident with the plane.

If two planes are incident with a point, any line incident with each of them is incident with the point.

The terms pencil and bundle, which were used of the elements of chapter I, will now be applied in an analogous way to the projective elements.

Def. 24. The set of all lines incident at once with any plane $\alpha$ and any point $A$ (incident with $\alpha$ ) is called a pencil of lines.

The set of all points incident with a line $a$ is called a pencil of points.

The set of all planes incident with a line $a$ is called a pencil of planes or axial pencil.

Pencils of lines, points, and planes are called one-dimensional forms or forms of the first grade.

DEF. 25. A two-dimensional form or form of the second grade is one of the following:

1. The set of all lines incident with a point.

2. The set of all lines incident with a plane.

3. The set of all planes incident with a point.

4. The set of all points incident with a plane.

1 and 3 are also called bundles of lines and bundles of planes. The system of all points and lines incident with a plane is called a plane system or field; and the set of all planes and lines incident with a point is called a point system or point field.

DEF. 26. The set of all points in space and the set of all planes in space are called three dimensional forms or forms of the third grade. The set of all points and planes in space is the space system or space field.

DEF. 27. A figure is any set of points, lines, and planes.

DeF. 28. If $A$ is a point incident with no point of a figure $w$ (consisting of points and lines) the system of all elements at once incident with $A$ and the elements of $w$ is the projection of $w$ from $A . \quad A$ is called a center of perspectivity. If $w^{\prime}$ is a figure consisting of lines and planes, and $a($ or $x)$ a line (or plane) not incident with any element of $w^{\prime}$, then the system of all elements at once incident with $a$ (or $\alpha$ ) and $w^{\prime}$ is the section of $w^{\prime}$ by $a$ (or $\alpha$ ). If two figures have a projection or section in common, or if one is a section of the other, they are said to be perspective figures.

DEF. 29. If $1,2,3, \ldots$ are elements of a one-dimensional form, and $1^{\prime}, 2^{\prime}, 3^{\prime}, \ldots$ are elements of another one-dimensional form, perspective with $1,2,3, \cdots$, we write $(1,2,3, \cdots) \approx\left(1^{\prime}, 2^{\prime}, 3^{\prime}, \cdots\right)$. If

$$
(1,2,3, \cdots) \bar{\pi}\left(1^{\prime}, 2^{\prime}, 3^{\prime}, \cdots\right) \cdot \bar{\pi}\left(1^{n}, 2^{n}, 3^{n}, \cdots\right)(n=2,3, \cdots),
$$


we say that the figures $(1,2,3,4, \ldots)$ and $\left(1^{n}, 2^{n}, 3^{n}, 4^{n}, \ldots\right)$ are projective, and use the notation $(1,2,3,4, \cdots) \pi\left(1^{n}, 2^{n}, 3^{n}, 4^{n}, \cdots\right)$.

\section{§ 3. Order and continuity of projective elements.}

DEF. 30. A finite or infinite set of collinear points are in the order $A \ldots B \ldots C \ldots K$ if they are projected from a proper point $O$ by lines in the order $a \ldots b \ldots c \cdots k$ (see chapter $\mathrm{I}, \S 4$ ).

Lemma $a$. This definition is independent of the choice of $O$.

Lemma $b$. If two sets of points $A, B, C, \ldots$ and $A^{\prime} B^{\prime} C^{\prime} \cdots$ are sections of the same (one-dimensional) pencil of lines in such a way that $A, A^{\prime}, B, B^{\prime}$, etc., are incident with the same line of the pencil, then if one set is in the order $A \ldots B \ldots C \ldots K$, the other set is in the order $A^{\prime} \ldots B^{\prime} \ldots C^{\prime} \ldots K^{\prime}$.

Lemma $c$. If two ranges $l_{1}$ and $l_{2}$ are sections of a pencil of planes $S$ and the points $A_{1}$ and $A_{2}, B_{1}$ and $B_{2}$, etc., are incident with the same plane, then if the order of a set of points on $l_{1}$ is $A_{1} \cdots B_{1} \cdots C_{1} \cdots K_{1}$, the order of the corresponding set on $l_{2}$ is $A_{2} \ldots B_{2} \ldots C_{2} \ldots K_{2}$.

DEF. 31. A set of elements of any form of the first grade (not a pencil of points) are in the order $\cdots l \cdots n \cdots n \cdots k \cdots$ if their section by a line is a set of points in the order $\ldots L \ldots M \ldots N \ldots K \ldots$ If four elements are in the order 1234,1 and 3 are said to separate 2 and 4 .

TH. 56 (I-X). If 123 are any three distinct elements of a one-dimensional form, there exists an element 4 in the order 1234 . If elements $1,2,3,4$ are in the order 1234 they are distinct. The notation for the order of a set of elements $\ldots l \cdots n \ldots m \ldots k \cdots$ of a one-dimensional form can be permuted cyclically or reversed. No other permutation is possible. From $l \cdots n \cdots m \cdots k$ and $l n k p$ follows $l \cdots n \cdots m \cdots k p$. From 1234 and $1235(4 \neq 5)$ follows either 1245 or 1254 . Any set of elements $\ldots l^{\prime} \ldots k^{\prime} \ldots$ perspective or projective with a set in the order $\ldots l \ldots n \ldots m \ldots k \ldots$ in such a way that $l^{\prime}$ corresponds to $l$, etc., are in the order $\ldots l^{\prime} \ldots n^{\prime} \ldots m^{\prime} \ldots k^{\prime} \ldots$.

TH. 57 (I-XI). If all the elements of a one-dimensional form consist of two sets, such that each set consists of at least two elements, and no two elements of one set separate two elements of the other set, then there exist two elements $p$ and $p^{\prime}$ that separate each element (distinct from $p$ and $p^{\prime}$ ) of one set from each element (distinct from $p$ and $p^{\prime}$ ) of the other set.

\section{§4. Principle of duality.}

All the theorems of projective geometry can be deduced from theorems 5157. These theorems remain entirely unchanged if the words point and plane are interchanged. Hence anything that can be deduced from them about points 
can be deduced also about planes. Hence we have, referring to a proposition deducible from theorems $51-57$ as a projective proposition :

TH. 58 (I-XI). Fundamental theorem of duality. Any valid projective proposition remains valid if the words point and plane are interchanged in its complete statement.

From this duality of space follows a like duality among the planes and lines or points and lines of any point- or plane-field. If $\pi$ is any plane, any theorem about the incidence and order of the lines and points in $\pi$ will hold for the incidence and order of the planes and lines obtained by projecting the lines and points of $\pi$ from a point $O$. The space dual of this latter theorem is a theorem about points and lines, in any plane $\sigma$; or in particular about the plane $\sigma=\pi$. Hence we have :

Tн. 59 (I-XI). Any valid projective proposition about points and lines incident with a plane $\pi$ remains valid if the words point and line are interchanged.

As the space dual of theorem 59 we have:

TH. 60 (I-XI). Any valid projective proposition about lines and planes incident

with a point $P$ remains valid if the words plane and line are interchanged.

The general term incidence has been used in this section to bring out the duality of space in a formal way. In the following sections, however, we shall use the words intersect, lie in, are on, etc., according to the ordinary usage.

\section{§5. Harmonic conjugates.}

The following theorems are well-known and are satisfactorily proved in the standard text-books.

Tн. 61 (I-X). If $A, B, C$ are three points of a line $l$, and $A^{\prime}, B^{\prime}, C^{\prime}$ are any three points of any other line $l^{\prime}, A B C$ can be projected to $A^{\prime} B^{\prime} C^{\prime}$ by the use of two centers of perspectivity.

Corollary. If $A, B, C$, and $A^{\prime}, B^{\prime}, C^{\prime}$ are on the same line $l$, three centers of perspectivity are sufficient.

Tн. 62 (I-X). If $A, B, C, D$ are any four points of a line,

$$
(A B C D) \pi(B A D C) \pi(C D A B) \text {. }
$$

DEF. 32. The figure consisting of three non-collinear points and the three lines incident with them by pairs is called a triangle.

DEF. 33. The figure consisting of four complanar points (no three of which are collinear) and the six lines incident with them by pairs is called a complete quadrangle. The four points are called the vertices; the six lines, the sides; two sides that do not have a vertex in common are opposite sides; and the points of intersection of opposite sides are diagonal points. The triangle of the diagonal points is called the diagonal triangle.

DEF. 34. The plane dual of the above figure is a complete quadrilateral. It has four sides, six vertices, and three diagonal lines, etc. 
Tн. 63 (I-X). Let $A B C$ and $A^{\prime} B^{\prime} C^{\prime}$ be two triangles lying in the same or in different planes. If the joins of corresponding vertices, $A A^{\prime}, B B^{\prime}$, $C C^{\prime}$, meet in a point, the corresponding sides, $A B$ with $A^{\prime} B^{\prime}, B C$ with $B^{\prime} C^{\prime}, A C$ with $A^{\prime} C^{\prime}$, intersect in three points of a line. Conversely, if the intersections of corresponding sides lie on a line the joins of corresponding vertices meet in a point.

TH. 64 (1-X). If five sides of one complete quadrangle intersect five sides of another complete quadrangle (no point of intersection being a vertex) in collinear points, then the point of intersection of the sixth side of one with the sixth side of the other lies on the same line with the other five intersection points.

Def. 35. If $A$ and $C$ are diagonal points of a quadrangle, and $B$ and $D$ the intersections of the remaining pair of opposite sides with the line $A C, D$ is called the fourth harmonic or harmonic conjugate of $B$ with respect to $A$ and $C$.

TH. 65 (I-X). If $D$ is the harmonic conjugate of $B$ with respect to $A$ and $C$, there is only one such point $D, B$ is the harmonic conjugate of $D$ with respect to $A$ and $C$, and the points $A, C$ are separated by $B, D$.

DEF. 36. If $D$ is the fourth harmonic of $B$ with respect to $A$ and $C$, the pair $B D$ are said to separate $A C$ harmonically ; $A B C D$ are sometimes said to be four harmonic points.

TH. 66 (I-X). If $(A B C D) \pi\left(A^{\prime} B^{\prime} C^{\prime} D^{\prime}\right)$ and the pair $B D$ separates the pair $A C$ harmonically, then $B^{\prime} D^{\prime}$ separates $A^{\prime} C^{\prime}$ harmonically. Conversely, if $B$ and $D$ separate $A$ and $C$ harmonically, and $A^{\prime}, B^{\prime}, C^{\prime}, D^{\prime}$ are any four points such that $B^{\prime}, D^{\prime}$ separate $A^{\prime}, C^{\prime}$ harmonically, then $(A B C D) \pi\left(A^{\prime} B^{\prime} C^{\prime} D^{\prime}\right)$. If $B$ and $D$ separate $A$ and $C$ harmonically, then $A$ and $C$ separate $B$ and $D$ harmonically.

Corollary. If $A B C D$ are four harmonic points, besides the perspectivities of theorem $62(A B C D) \pi(A D C B) \pi(C B A D)$.

\section{$\S 6$. The fundamental theorem of projective geometry.}

DEF. 37. If $A, B, C$ are any three points of a line, a point $D$ is said to be harmonically related to $A, B, C$ if it is any one of a finite set of points $D_{1} \ldots D_{n}(n \geqq 1)$ such that $D_{1}$ is the fourth harmonic of one of $A, B, C$ with respect to the other two and $D_{k}(k=2 \cdots n)$ the fourth harmonic of one of the set $A B C D_{1} \ldots D_{k-1}$ with respect to two others of the set.

TH. 67 (I-XI). If $A, B, C$ are any three points of a line and $P$ and $Q$ any two other points of the same line then there are two points $D$ and $E$ which at the same time are harmonically related to $A, B, C$ and separate $P$ and $Q . *$

* F. KLeIN (Mathematische Annalen, vol. 6 (1873) p. 139) first pointed out that von STAUDT's proof of the fundamental theorem was incomplete without the use of some such axiom ás $\mathrm{XI}$. 
Tн. 68 (I-XI). The findamental theorem. If $A B C D$ are four collinear points and $(A B C D) \pi\left(A^{\prime} B^{\prime} C^{\prime} D^{\prime}\right)$ then by any process of projection and section for which $(A B C D) \pi\left(A^{\prime} B^{\prime} C^{\prime} D^{\prime \prime}\right), D^{\prime}=D^{\prime \prime}$.*

TH. 69 (I-XI) If $A, B$ and $C, D$ are two pairs of points that do not separate one another then there exists one and only one pair $P, Q$ that at once harmonically separates $A, B$ and $C, D$.

By means of this theorem it is seen that any one to one correspondence preserving harmonic relations also preserves order and then by theorem 67 and the principle of duality we obtain the following general form of the "fundamental theorem."

DEF. 38. A one-to-one correspondence between two forms (or of a form with itself) is called projective if, whenever elements $1,2,3, \ldots$ of the same one-dimensional form correspond to elements $1^{\prime}, 2^{\prime}, 3^{\prime}, \ldots$,

$$
(123 \cdots) \pi\left(1^{\prime} 2^{\prime} 3^{\prime} \cdots\right) \text {. }
$$

TH. 70 (I-XI). A more general fundamental theorem. Any one-to-one correspondence between two one-dimensional forms by which every four harmonic elements of one form correspond to four harmonic elements of the other form, is projective.

Tн. 71 (I-XI). A one-to-one correspondence between two forms $I$ and $I^{\prime}$ (where I may be the same as $I^{\prime}$ ) of the same grade higher than the first is projective if every one-dimensional form of elements of I corresponds to a to a one-dimensional form of $\mathrm{I}^{\prime}$, and reciprocally.

DEF. 39. A projective correspondence by which points correspond to points is a collineation. A projective correspondence by which elements of one kind correspond to elements of another kind is called polar.

\section{\$7. Involution and polar system.}

DEF. 40. An involution is such a projective correspondence of a one-dimensional form with itself that if an element 1 corresponds to $1^{\prime}$ then $1^{\prime}$ corresponds to 1 . Pairs of elements such as 1 and $1^{\prime}$ are called pairs of conjugate elements. The following theorems stated for pencils of points apply by duality to involutions in general.

TH. 72 (I-XI). An involution has either two or no self-corresponding points.

M. Pasch (Vorlesungen über Neuere Geometrie, 8, 15, Leipzig, 1882) gave a proof which made use of congruence axioms and the so-called Archimedean axiom.

H. Wiener (Ueber die Grundlagen und den Aufbau der Geometrie, Jahresbericht der Deutschen Mathematikervereinigung, vol. 1, p. 47, vol. 3, p. 70) and Schur (Mathe matische Annalen, vol. 51 (1898), p. 401, vol. 55 (1902), p. 265) have shown that it can be demonstrated by congruence axioms alone without the Archinedean axiom.

L. BAIser (Mathematische Annalen, vol. 55 (1902), p. 293) has given a proof based upon hypotheses equivalent to ours. 
Corollary. If an involution has two fixed points, $A, B$, they separate harmonically every pair of corresponding points.

Tн. 73 (I-XI). If $A, A^{\prime}, B, B^{\prime}$ are arbitrary points of a line, there exists one and only one involution in which $A$ and $A^{\prime}, B$ and $B^{\prime}$ are conjugate points.

Corollary. The theorem is true also if $A=A^{\prime}$ or $B=B^{\prime}$, or both.

Tн. 74 (I-XI). The three pairs of opposite sides of a complete quadrangle QRST are cut in three pairs of an involution by any straight line which lies in the plane of the quadrangle and passes through none of its vertices.

Tн. 75 (I-XI). If two pairs, $A A^{\prime}, B B^{\prime}$, of an involution do not separate each other, no two pairs of the involution separate each other, and the involution has two fixed points. If two pairs, $A A^{\prime}, B B^{\prime}$, separate each other, every two pairs of the involution separate each other, and the involution has no fixed point.

DeF. 41. An involution with two fixed points is called hyperbolic. An involution with no fixed point is called elliptic.

Tн. 76 (I-XI). If $B$ and $C$ are two points of a line, there is one, and only one, pair of a given elliptic involution that separates $B$ and $C$ harmonically.

TH. 77 (I-XI). If two pencils are projective, an involution in one corresponds to an involution in the other.

DEF. 42. The points and lines of a plane constitute a polar system if they are in such a reciprocal one to one correspondence that to the pine incident with any two ${ }_{\text {points }}^{\text {lines }}$ corresponds the ${ }_{\text {point }}^{\text {line }}$ incident with the corresponding pair of

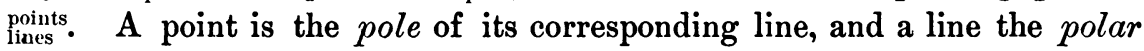
of its corresponding point. A polar system is elliptic if no element is incident with its corresponding element; it is hyperbolic if some element is incident with its corresponding element. $\quad \mathrm{A} \underset{\text { point } L^{\prime}}{\text { line } l^{\prime}}$ is conjugate to a ${ }_{\text {point } L}^{\text {line }} L$ if it passes through the ${ }_{\text {polar }}^{\text {pole }}{ }_{L}^{l}$.

The proofs of the existence of the various collineation and polar systems depend on constructions that are well-known and for which it is easily verified that our hypotheses are sufficient. See

J. SteInER: Synthetische Geometrie, Die Theorie der Kegelschnitte, Leipzig, 1887.

H. Schroeter : Theorie der Oberflächen Ziveiter Ordnung, Leipzig, 1880.

K. G. C. von Staudt: Beiträge zur Geometrie der Lage, Nürnberg, 1856.

In particular, in SteIneR's Synthetische Geometrie on pp. 411-414 and 422424 will be found the proof of the existence of elliptic polar systems.

In the following section we outline the definition and deduction of metric euclidean geometry. For that argument we employ the following theorems : 


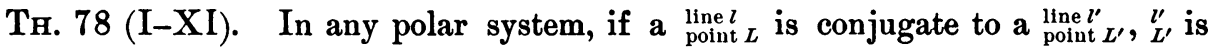
conjugate to ${ }_{L}^{l}$. The system of all such pairs of conjugate lines $_{\text {points }}$ of a given point form an involution. There exist polar systems in which all these involutions are elliptic. A polar system is fully determined by the involutions upon a pair of conjugate points $_{\text {lines }}$.

TH. 79 (I-XI). If $A_{1} A_{2} A_{3}$ are the vertices of a triangle and $P_{1} P_{2} P_{3}$ the points of intersection of the lines $A_{2} A_{3}, A_{3} A_{1}, A_{1} A_{2}$ respectively with a line $p$, and $M_{1} M_{2} M_{3}$ the harmonic conjugates of $P_{1} P_{2} P_{3}$ with respect to $A_{2} A_{3}, A_{3} A_{1}, A_{1} A_{2}$ then the three lines joining $M_{1} M_{2} M_{3}$ to the three conjugate points of $P_{1} P_{2} P_{3}$ in any involution on $p$, meet in a point.

The metric special case of this theorem is that the perpendiculars at the middle points of the sides of a triangle meet in a point. For its proof one has only to consider the complete quadrangle $P . M_{1} M_{2} M_{3}$ where $P$ is the intersection of $P_{1}^{\prime} M_{1}$ with $P_{2}^{\prime} M_{2}, P_{1}^{\prime}$ and $P_{2}^{\prime}$ being the conjugate points of $P_{1}$ and $P_{2}$. It leads at once to the following

TH. 80 (I-XI). Let $\Sigma$ be an elliptic polar system and $A_{1} A_{2} A_{3}$ any triangle and $M_{1} P_{1}, M_{2} P_{2}$ the unique pairs of conjugate points that separate $A_{2} A_{3}$ and $A_{3} A_{1}$ harmonically. If $P_{3}$ is the point of intersection of $P_{1} P_{2}$ (or of $M_{1} M_{2}$ ) with $A_{1} A_{2}$ then the fourth harmonic $M_{3}$ of $P_{3}$ with respect to $A_{1} A_{2}$ is also its conjugate with respect to $\Sigma$. Furthermore the three conjugate lines of the sides of the triangle at $M_{1}, M_{2}, M_{3}$ meet in a point $P$, the pole of $P_{1} P_{2}$.

\section{§ 7. Similar figures.*}

DeF. 43. In this section the term projective transformation will be used as synonymous with collineation. If a point $X$ corresponds to a point $X^{\prime}$ in a collineation, $X$ is said to be projectively transformed into or to go into, $X^{\prime}$. A reflection of the points of a line by a pair of points of the line $A A^{\prime}$ is a projective transformation by which each point of the line goes into its fourth harmonic with respect to $A$ and $A^{\prime}$; i. e., the pairs of corresponding points form an hyperbolic involution; For this transformation we use the notation $\left(A A^{\prime}\right)$.

A reflection of the points of a $a_{\text {space }}^{\text {plane }}$ by the (non-incident) point $A$ and $\operatorname{line}_{\text {plane } a}$ of the plane $_{\text {space }}$ is a projective transformation such that each point $X$ of

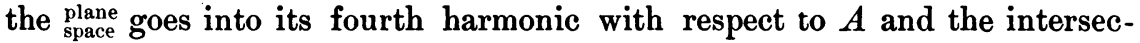
tion of $A X$ with $_{a}^{a}$. We use the notation ${ }_{(A)}^{(A a)}$.

If by one projective transformation, $\mu$, points $A, B, C, D, \ldots$ go into $A^{\prime} B^{\prime} C^{\prime} D^{\prime} \ldots$ and by another, $\nu, A^{\prime} B^{\prime} C^{\prime} D^{\prime} \ldots$ go into $A^{\prime \prime} B^{\prime \prime} C^{\prime \prime} D^{\prime \prime} \ldots$, then the correspondence of $A B C D \ldots$ with $A^{\prime \prime} B^{\prime \prime} C^{\prime \prime} D^{\prime \prime} \ldots$ is evidently projective; it is called the product of $\nu$ and $\mu$ and written $\nu \mu$. We write $\mu A=A^{\prime}$ and thus

* For references see footnote, $\dagger \dagger, \S 1$, chap. I.

Am. Trans. Math. Soc. 23 
have $\nu(\mu A)=\nu A^{\prime}=A^{\prime \prime}=(\nu \mu) A$. Further $\rho(\nu \mu)=(\rho \nu) \mu$ and if $\sigma$ is any reflection $\sigma^{2}$ is the identity. We denote the identity by $\omega$ - thus $\sigma^{2}=\omega$.

If a transformation is such that every pair of conjugate elements of an involution or polar system, $\Sigma$, goes into the same or another conjugate pair, the transformation is said to leave $\Sigma$ invariant.

Tн. 81 (I-XI).* Any collineation that leaves invariant an elliptic involution or a (plane) elliptic polar system is either a reflection by a pair of corresponding elements or a product of such reflections. A reflection by any pair of an elliptic involution or polar system leaves the involution or polar system invariant. By a suitably chosen transformation leaving the system invariant, any pair of incident elements of the system can be transformed into any other pair of incident elements of the same kind.

Combining results of this theorem with the theory of parallel lines ( $\S 6$, chapter II) we are able to give a definition of congruent angles, and thus to establish the theory of similar figures. It results immediately from theorem 43 (the strong parallel proposition) that the improper projective points constitute an improper plane, whereas every other projective point is a bundle of lines, all passing through a point in the sense of chapter $I$. The improper plane (whose determination is unique by theorem 43 ) we call the plane at infinity. In this plane an elliptic polar system $\dagger \Sigma$ is arbitrarily chosen. $\Sigma$ determines at every point of space a polar system of planes and lines, of which a corresponding plane and line (or pair of conjugate lines or pair of conjugate planes) are said to be mutually perpendicular. In formal terms :

Def. 44. A plane $\alpha$ and line $a$ are mutually perpendicular if their intersections with the plane at infinity are a pair of corresponding elements $a^{\prime}$ and $A^{\prime}$ of $\Sigma$. Two intersecting lines are perpendicular if they meet the plane at infinity in conjugate points of $\Sigma$.

DeF. 45. One angle is congruent to another if the sides of the one can be transformed into the sides of the other by a collineation of space that leaves $\Sigma$ invariant. Since this relation is evidently mutual the angles are said to be congruent to one another.

The properties of congruent angles follow without difficulty from this definition and from theorem 81 . This may be conveniently verified by comparison with HILBERT's axioms IV 4 and IV 5.

Our axioms and definitions are therefore sufficient to establish the theory of similar figures.

* There is, of course, an analogous theorem for space polar systems.

$\dagger$ "The imaginary circle at infinity." That the choice of $\Sigma$ is arbitrary is one of the important properties of space; one tends to overlook this if congruence is introduced by axioms. 


\section{§8. Congruent segments.}

To define congruent segments we have only to specify a subgroup of the group of similarity collineations of space that leave $\Sigma$ (and hence, of course, the plane at infinity) invariant. A perpendicular reflection or more simply (since we shall no longer deal with other reflections) a reflection, by a proper plane $\alpha$, is the reflection $(A \alpha)$ where $A$ is the correspondent in $\Sigma$ of the line $a$ in which $\alpha$ meets the plane at infinity.

DeF. 46. A segment $A B$ is congruent to a segment $A^{\prime} B^{\prime}$ if $A B$ can be transformed into $A^{\prime} B^{\prime}$ by a finite number of reflections. Since this relation is evidently mutual the segments are said to be congruent to one another.

That the usual properties of congruent segments can be deduced from this definition is obvious on comparison with HILBERT's axioms IV 1, 2, 3, 6, as soon as one establishes, by the aid of thorems 79 and 80 :

Tr. 82 (I-XII). $O A$ is the only segment with end-point $O$, in the half-line $O A$, that is congruent to $O A$.

\section{§ 9. The system of axioms is categorical.}

The metric properties of space having been established as indicated in $\S 8$, the usual definitions can be given for the length of a segment in terms of a unit segment.

Coördinate axes can now be introduced in the usual way: namely, a unit of length, and three non-complanar lines through a point $O$ are chosen arbitrarily. On each line there is thus established a correspondence between the points and the real numbers, positive and negative. The coördinates of a point $P$ are defined as the numbers associated with the points in which three planes through the point $P$ and parallel to the three pairs of coördinate lines meet the corresponding three coördinate lines.

Tн. 83 (I-XII). To every set of three coördinates $(x, y, z)$ corresponds a point, and to every point corresponds a set of three coördinates. The necessary and sufficient condition that three distinct points, $P_{1}, P_{2}, P_{3}$, whose coördinates are respectively $\left(x_{1}, y_{1}, z_{1}\right),\left(x_{2}, y_{2}, z_{2}\right),\left(x_{3}, y_{3}, z_{3}\right)$ are in the order $P_{1} P_{2} P_{3}$, is the existence of a number $k$ such that $0<k<1$, and every one of the following fractions, not of the form $\frac{0}{0}$, is equal to $k$.

$$
\begin{array}{lll}
x_{1}-x_{2} & y_{1}-y_{2} \\
x_{1}-x_{3} & y_{1}-y_{3}
\end{array} \quad \frac{z_{1}-z_{2}}{z_{1}-z_{3}} .
$$

TH. 84 (I-XII). If $K$ and $K^{\prime}$ are any two classes that verify axioms I-XII, then any proposition stated in terms of points and order that is valid of the class $K$ is valid of the class $K^{\prime}$. 
Proof. In $K$ and in $K^{\prime}$ introduce a system of coördinates; let a point in one class correspond to a point in the other class whose coördinates are the same three numbers. By theorem 83, if three points of $K$ are in a given order, the corresponding three points of $K^{\prime}$ are in the same order. 